(C) 2003 International Press

Adv. Theor. Math. Phys. 7 (2003) 331-367

\title{
A New Approach to Quantising Space-Time: I. Quantising on a General Category
}

\author{
C.J. Isham \\ The Blackett Laboratory \\ Imperial College of Science, Technology \& Medicine \\ South Kensington \\ London SW7 2BZ \\ c.isham@imperial.ac.uk
}

\begin{abstract}
A new approach is suggested to the problem of quantising causal sets, or topologies, or other such models for space-time (or space). The starting point is the observation that entities of this type can be regarded as objects in a category whose arrows are structure-preserving maps. This motivates investigating the general problem of quantising a system whose 'configuration space' (or history-theory analogue) can be regarded as the set of objects $\mathrm{Ob}(\mathcal{Q})$ in a category $\mathcal{Q}$. In this first of a series of papers, we study this question in general and develop a scheme based on constructing an analogue of the group that is used in the canonical quantisation of a system whose configuration space is a manifold $Q \simeq G / H$ where $G$ and $H$ are Lie groups. In particular, we choose as the analogue of $G$ the monoid of 'arrow fields' on $\mathcal{Q}$. Physically, this means that an arrow between two objects in the category is viewed as some sort of analogue of momentum. After finding the 'category quantisation monoid', we show how suitable representations can be constructed using a bundle of Hilbert spaces over $\mathrm{Ob}(\mathcal{Q})$.
\end{abstract}

e-print archive: http://lanl.arXiv.org/abs/gr-qc/0303060 


\section{Introduction}

One of the enduringly, and endearingly, fascinating challenges in quantum gravity is to give meaning to the idea of quantising space, or space-time, at a level that is more fundamental than that of quantising a metric tensor on a background manifold. ${ }^{1}$ For example, one comes across phrases in the literature such as 'quantising causal sets ${ }^{2}$, [1] [2] [3] [4], or 'quantising topology', and the goal of the present paper is to invest these concepts with a new, and precise, meaning.

In the present paper an operator-based approach to quantising space, or space-time, structure is described. The ensuing theory is applicable to two types of physical situation. The first is 'canonical quantisation', where the states in the Hilbert space refer to the situation at a fixed time. For example, in a theory of the quantisation of the topology $\tau$ of physical space, the states might be functions $\tau \mapsto \psi(\tau)$, or an extension thereof. Such a theory would then need to be augmented with a 'Hamiltonian' operator to specify how these states evolve in (possibly, a discrete) time.

On the other hand, it is not appropriate to talk about the canonical quantisation of causal sets since each causal set $c$ is a complete space-time in itself, and hence a state function $c \mapsto \psi(c)$ has no physical meaning within the interpretative framework of standard quantum theory. However, states of this type are meaningful in a consistent-history approach to quantum theory. More precisely, in the 'HPO' (history projection operator) method, propositions about complete histories of a system are represented by projection operators on a 'history Hilbert space' [5]. In the case of causal sets, the propositions would include statements about the causal-set structure of space-time. In a theory of this type, the analogue of 'dynamics' is coded into the decoherence functional that is to be constructed from the basic quantum operators in the history Hilbert space.

It is important to keep in mind these two different ways of using operators and Hilbert spaces: (i) a canonical quantum theory, and (ii) the HPO approach to a consistent history theory. The general mathematical framework is the same in both cases, but the physical interpretation is quite different.

Let us now consider in more detail the construction a quantum history

\footnotetext{
${ }^{1}$ This is not meant to imply that it is obvious how to quantise a metric field on a background space or space-time: it is not!

${ }^{2} \mathrm{~A}$ 'causal set' is a partially-ordered set $P$ whose elements represent points in a discrete model for space-time. If $p, q \in P$ are such that $p \leq q$ then the physical interpretation is that an event at $p$ can causally influence events at $q$.
} 
theory of causal sets. A first guess might be that the history state vectors are functions $c \mapsto \psi(c)$, or some generalisation thereof. This sounds plausible, but how is it to be justified; and what is the appropriate 'generalisation' of a function $\psi$ of causal sets?

In the canonical quantisation of, say, a particle moving in one dimension, the rationale for identifying states with wave functions in the Hilbert space $L^{2}(\mathbb{R})$ lies in the existence of operators $\hat{x}$ and $\hat{p}$ that are assumed to satisfy the canonical commutation relations $[\hat{x}, \hat{p}]=i \hbar$. It then follows from the famous theorem of Stone and Von Neumann that any irreducible representation of this canonical algebra ${ }^{3}$ is unitarily equivalent to the familiar one on $L^{2}(\mathbb{R})$ in which $(\hat{x} \psi)(x):=x \psi(x)$ and $(\hat{p} \psi)(x):=-i \hbar d \psi / d x$.

More generally, consider a system whose configuration space is a finitedimensional differentiable manifold $Q$ such that $Q \simeq G / H$, where $G$ and $H$ are Lie groups. The analogue of the canonical commutation relations includes a representation of the Lie algebra of $G$, and the elements of this algebra are the momentum variables in the theory. The question arises, therefore, of whether there is analogue of momentum for causal sets. When $Q \simeq G / H$, the Lie group $G$ generates transformations from one point in $Q$ to another, which leads us to consider how one causal set can be 'transformed' into another ${ }^{4}$. Similarly, in quantum topology, we would seek a natural way of 'transforming' from one topological space to another.

In the case of causal sets, one can imagine trying to remove, or add, points and links, but it is not easy to describe a general scheme for doing this. For example, removing a point (and the associated links) might result in a disconnected causal set. But suppose we do not wish to admit spacetimes of this type: what then? Similarly, adjoining a point to a causal set is not trivial since enough links must be also added to ensure that the resulting structure is a partially-ordered set, and this can be done in different ways.

The key idea of the present paper is that, in the example of causal sets, what 'connects' one causal set to another is the collection of all orderpreserving maps between them. This suggests that to each such map $f: c_{1} \rightarrow$ $c_{2}$ there is to be associated an operator $\hat{d}(f)$. Moreover, if we have three causal sets $c_{1}, c_{2}, c_{3}$, and order-preserving maps $f: c_{1} \rightarrow c_{2}$, and $g: c_{2} \rightarrow c_{3}$, then the composition $g \circ f: c_{1} \rightarrow c_{3}$ is also order-preserving. It is natural to postulate that the operators $\hat{d}(f)$ reflect this structure by satisfying the

\footnotetext{
${ }^{3}$ More precisely, the theorem refers to weakly continuous representations of the exponentiated form of the canonical commutation relations.

${ }^{4}$ This question has also been of particular interest to Rafael Sorkin and Ioannis Raptis in the context of their own work on causal sets; private communication.
} 
relations

$$
\hat{d}(g \circ f)=\hat{d}(g) \hat{d}(f)
$$

or, perhaps,

$$
\hat{d}(g \circ f)=\hat{d}(f) \hat{d}(g),
$$

since, at this stage, there is no prima facie reason for preferring any particular ordering of the operators.

Note that in a scheme of this type it is easy to use just connected causal sets, if so desired. Or we can require the causal sets to be finite; or restrict our attention to order-preserving maps that are one-to-one; or to any of a number of variants of the basic idea if there is some good physical reason for doing so. It is clear that a similar idea could be applied to topological spaces, with the analogue of order-preserving maps being continuous functions.

These preliminary ideas could be developed into genuine quantisation schemes for causal sets and topological spaces. However, these particular examples admit a natural generalisation that applies to many different physical situations, and it is this generalisation that is described in the present paper. The application of this scheme to causal sets is discussed in a companion paper [7].

To motivate this generalisation, consider the example of, say, finite causal sets. The key remark is that these can be viewed as the objects of a category, whose arrows/morphisms are order-preserving maps. Similarly, one can imagine forming a category whose objects are topological spaces of some specific physical interest, and whose arrows are continuous maps.

Thus we are led to the following general problem. Namely, to construct the quantum theory of a system whose 'configuration space' (or history analogue) is the set of objects in some category $\mathcal{Q}$, and in which the role of momentum transformations is played by the arrows in $\mathcal{Q}$. More precisely, if $f: A \rightarrow B$ is an arrow (i.e., the objects $A$ and $B$ are the domain and range of $f$ respectively), then we think of $f$ as providing a (partial) description of how to 'transform' from $A$ to $B$. In general, there will be many arrows from $A$ to $B$, and we shall regard the set of all of them, denoted $\operatorname{Hom}(A, B)$, as affording the complete description of how to transform from $A$ to $B$.

Note that for this idea to be mathematically meaningful, the category $\mathcal{Q}$ must be 'small' in the sense that the collection of all objects, $\mathrm{Ob}(\mathcal{Q})$, in $\mathcal{Q}$, and the collection of all arrows, $\operatorname{Hom}(\mathcal{Q})$, in $\mathcal{Q}$ must be genuine sets, not classes. For example, the category of all sets is certainly not of this type. 
To construct a quantum theory on a general small category $\mathcal{Q}$ we generalise what was said above for causal sets. Thus we expect each arrow $f \in \operatorname{Hom}(\mathcal{Q})$ to be associated with an operator $\hat{d}(f)$ in such a way as to represent the law of arrow composition by the operator relations Eq. (1) (or Eq. $(2)$ ). If the objects in $\mathrm{Ob}(\mathcal{Q})$ have a physically important internal structureas manifested mathematically by the sets $\operatorname{Hom}(A, A), A \in \mathrm{Ob}(\mathcal{Q})$, being non-trivial - this should be reflected in the quantum theory. In particular, we anticipate that the (history) state vectors are vector-space valued functions on $\mathrm{Ob}(\mathcal{Q})$, in analogy to what happens for a manifold $Q \simeq G / H$ where, generically, the states are cross-sections of a vector bundle over $Q$ whose fibre carries a representation of $H$. In fact, as we shall see, the construction of a quantum theory on $\mathcal{Q}$ involves a generalisation of the idea of vector-valued functions.

A variety of physically interesting situations are special cases of this categorical scheme. For example:

1. $\mathcal{Q}$ is a category of finite (perhaps connected) causal sets interpreted as a history theory. Another possibility is to use causal sets that are locally finite. Or one could choose some 'master' causal set ${ }^{5} U$ and let $\mathcal{Q}$ be the category of all causal subsets of $U$, with the arrows being the order-preserving embeddings of one causal set in another.

2. $\mathcal{Q}$ is a small category of partially ordered sets interpreted canonically as the structure of physical space at a given 'time'.

3. $\mathcal{Q}$ is a small category of topological spaces. This gives a new approach to 'quantum topology': to be interpreted as a history theory if the objects represent space-time, and as a canonical theory if the objects represent space. ${ }^{6}$

4. $\mathcal{Q}$ is a small category of differentiable manifolds, with the arrows being differentiable maps between manifolds, regarded as models of either space-time or space.

5. A more bizarre example is to take $\mathcal{Q}$ to be a small category of groups, with the arrows being group homomorphisms. For example, perhaps the symmetry group of a unified field theory undergoes 'quantum fluctuations' near the big bang singularity? This certainly gives a novel interpretation to the idea of "quantum group theory":-)

\footnotetext{
${ }^{5}$ Fay Dowker: private discussion.

${ }^{6}$ More generally, $\mathcal{Q}$ could be a small category of locales (in the context of topology without points) whose arrows are localic maps.
} 
6. In all the examples above, the category $\mathcal{Q}$ is a category of sets with structure, and the arrows are maps that preserve this structure. Thus it is useful to look first at the case where $\mathcal{Q}$ is a small category of sets, and the arrows between two sets $A$ and $B$ are any functions from $A$ to $B$. This is studied in detail in [7].

7. An example of a category whose objects are not structured sets is a partially-ordered set (poset) $P$. The objects of this category are the points in $P$, and if $p, q \in P$, an arrow is defined to exist from $p$ to $q$ if and only if $p \leq q$ (hence there can be at most one arrow between any two points/objects). In this case, the objects have no internal structure, and so the quantum theory should be relatively simple.

This example is useful for providing a mathematically simple illustration of the general scheme, and we shall discuss it in [7]. However, it cannot be interpreted as a theory of quantum space-time (or space) of the type in which we are interested since each possible space-time (or space) is represented by structureless point ${ }^{7}$.

At this point, however, it is important to observe that there is an obvious problem with imposing Eq. (1) (or Eq. (2)) as it stands. Namely, the composition $g \circ f$ is only defined if the range, $\operatorname{Ran} f$, of $f$ is equal to the domain, Dom $g$, of $g$. Thus, if $f: A \rightarrow B$, and $g: C \rightarrow D$, the composition $g \circ f$ is only defined if $B=C$. On the other hand, the operator product $\hat{d}(g) \hat{d}(f)$ on the right hand side of Eq. (1) (or Eq. (2)) is always defined ${ }^{8}$. The resolution of this issue is one of the key steps in the quantum scheme. Two approaches are suggested: the first involves a semigroup $\operatorname{Sem}(\mathcal{Q})$ that is constructed from the arrows in $\mathcal{Q}$; the second, and preferred, method involves the idea of an 'arrow field'.

The plan of the paper is as follows. An initial approach to developing a quantum theory is discussed in Section 2. The focus is placed on equipping the set of arrows $\operatorname{Hom}(\mathcal{Q})$ of $\mathcal{Q}$ with a semigroup structure. We show how this semigroup, $\operatorname{Sem}(\mathcal{Q})$, generates transformations of the set of objects $\operatorname{Ob}(\mathcal{Q})$ : as such, it constitutes our first attempt at finding an analogue of the group $G$ used in the quantisation of a system whose configuration space is a manifold $Q \simeq G / H$.

\footnotetext{
${ }^{7}$ The canonical theory could be interpreted as that of a particle whose position is confined to one of the points in the poset $P$, but there is no obvious reason why such a system should be of any physical interest.

${ }^{8}$ If the operators $\hat{d}(f), f \in \operatorname{Hom}(\mathcal{Q})$, are unbounded then these products may not exist. But that is not the point at issue here which applies even when the operators concerned are all bounded.
} 
However, the construction of $\operatorname{Sem}(\mathcal{Q})$ is rather coarse, involving as it does the ad hoc introduction of an element ' $\star$ ' whose sole role is to serve as the value of $g \circ f$ when the range of $f$ does not equal the domain of $g$. This is remedied in Section 3 by the introduction of the idea of an 'arrow field', defined to be an assignment to each object $A \in \mathrm{Ob}(\mathcal{Q})$ of an arrow whose domain is $A$. The crucial property of the set $\operatorname{AF}(\mathcal{Q})$ of all arrow fields is that it has a natural semigroup structure without the need for additional, spurious elements.

The action of the semigroup $\operatorname{AF}(\mathcal{Q})$ on $\mathrm{Ob}(\mathcal{Q})$ is used in Section 4 to provide the foundation of the quantum scheme. We start in Section 4.2 with a simple approach in which the quantum states are complex-valued functions on $\mathrm{Ob}(\mathcal{Q})$. This suffices to construct the basic 'category quantisation monoid', each of whose faithful, irreducible representations is deemed to constitute a proper quantisation on $\mathcal{Q}$.

However, as it stands, this simple scheme is inadequate since the quantum operators do not distinguish arrows with the same domain and range. We solve this problem in Section 4.3.1 by generalising the state vectors to become vector-valued functions on the set of objects $\mathrm{Ob}(\mathcal{Q})$. It transpires that the vector space in which a function takes its values must vary from object to object, and each such 'multiplier' representation is associated with a presheaf of Hilbert spaces over $\mathrm{Ob}(\mathcal{Q})$. (However, no detailed language of presheaf theory is used in the present paper.)

The collection of basic quantum operators is completed in Section 4.4 with a computation of the adjoints of the operators $\hat{a}(X)$ that represent arrow fields $X \in \operatorname{AF}(\mathcal{Q})$. In Section 4.5, we compute the products $\hat{a}(X)^{\dagger} \hat{a}(X)$ and $\hat{a}(X) \hat{a}(X)^{\dagger}$ that might be expected to play an important role in specific applications of the quantum scheme. In Section 4.7 there are a few preliminary remarks about the irreducibility of the representations we have constructed. The paper concludes with Section 5, which is mainly a list of problems for further research.

The present paper, the first in a series, introduces the general theory of quantising on a category. In the second paper [7], the general theory is developed for the physically important case where $\mathcal{Q}$ is a category of sets. In [8] we return to the general theory and present an alternative approach in which state vectors are complex-valued functions on arrows, rather than, as in the present paper, on objects. Later papers in the series will describe further developments of some of the main ideas, and more concrete examples. 


\section{Quantising Using the $\operatorname{Semigroup} \operatorname{Sem}(\mathcal{Q})$}

\subsection{The Semigroup $\operatorname{Sem}(\mathcal{Q})$}

The key problem identified above in the context of Eq. (1) is that the composition $g \circ f$ of arrows is only defined if Ran $f=$ Dom $g$, whereas the operator product $\hat{d}(g) \hat{d}(f)$ (or $\hat{d}(f) \hat{d}(g)$ ) is always defined. In other words, the set $\operatorname{Hom}(\mathcal{Q})$ of arrows is only a partial semigroup under the law of composing arrows, whereas the (bounded) operators on a Hilbert space are a full semigroup under operator multiplication.

In this context, recall that a (full) semigroup is a non-empty set $S$ with a law of combination that is associative. Thus

$$
a(b c)=(a b) c
$$

for all $a, b, c \in S$. A semigroup with a unit element is called a monoid ${ }^{9}$. In a monoid (and unlike in a group) elements may not have inverses.

A partial semigroup $S$ is a more general structure in which not all pairs of elements $a, b \in S$ can be combined; if a pair $a, b$ can be combined, they are said to be compatible. The associativity law Eq. (3) is now imposed only when it makes sense: i.e., when the different elements in Eq. (3) are compatible in the appropriate way.

For our purposes, a key observation is that the equation Eq. (1), $\hat{d}(g \circ f)=$ $\hat{d}(g) \hat{d}(f)$, would be well defined if the elements $g$ and $f$ belonged to a full semigroup rather than only to a partial one. Thus, we start by considering if it is possible in general to convert a partial semigroup into a full semigroup.

One simple approach is to append an extra element $\star$ to a partial semigroup $S$, and then try to define a new combination law ' $\&$ ' by

$$
a \& b:= \begin{cases}a b & \text { if } a \text { and } b \text { are compatible } \\ \star & \text { otherwise }\end{cases}
$$

and

$$
\begin{aligned}
& \star \& a:=\star \\
& a \& \star:=\star \\
& \star \& \star:=\star
\end{aligned}
$$

\footnotetext{
${ }^{9}$ This distinction is not very important since a unit element can always be appended if one is not present.
} 
for all ${ }^{10} a \in S$. Note that it follows from these definitions that if $a$ and $b$ are compatible elements of $S$, then $a \& b \neq \star$.

The combination law ' $\&$ ' defined by Eqs. (4-7) on the extended set $S_{+}:=$ $S \cup\{\star\}$ has the serious failing that, in general, it is not associative. For example, consider $a, b, c \in S$ such that $a$ and $b$ are not compatible. Then, for all $c \in S$, we have $(a \& b) \& c=\star \& c=\star$. On the other hand, it could be that $b$ and $c$ are compatible, in which case $b \& c \neq \star$. Then, if $a$ is compatible with $b \& c$, which is possible, we have $a \&(b \& c) \neq \star$, and hence a failure of associativity.

However, this objection does not apply when the partial semigroup is the set of arrows in a category $\mathcal{Q}$, with $a \& b:=a \circ b$ for $a, b \in \operatorname{Hom}(\mathcal{Q})$. For $a$ and $b$ are not compatible if and only if $\operatorname{Ran} b \neq \operatorname{Dom} a$. But if $b$ and $c$ are compatible, then $b \& c=b \circ c$, and since Ran $b \circ c=$ Ran $b$ it follows that $a$ and $b \& c$ are not compatible.

In conclusion, the partial semigroup $\operatorname{Hom}(\mathcal{Q})$ of arrows in a small category $\mathcal{Q}$ can be given the structure of a full semigroup by augmenting the set $\operatorname{Hom}(\mathcal{Q})$ with an additional element $\star$ (which is not given a domain or range), and then defining, for all $f, g \in \operatorname{Hom}(\mathcal{Q})$,

$$
g \& f:= \begin{cases}g \circ f & \text { if Ran } f=\operatorname{Dom} g \\ \star & \text { otherwise }\end{cases}
$$

and

$$
\begin{aligned}
& \star \& f:=\star \\
& f \& \star:=\star \\
& \star \& \star:=\star
\end{aligned}
$$

for all $f \in \operatorname{Hom}(\mathcal{Q})$. This semigroup will be $\operatorname{denoted} \operatorname{Sem}(\mathcal{Q})$. It does not have a unit element. ${ }^{11}$

\subsection{An Embryo Quantum Theory on $\mathcal{Q}$}

If a Lie group $G$ acts on the left on a manifold $Q$ then an elementary (anti)representation of $G$ is given on the vector space of complex-valued functions

\footnotetext{
${ }^{10}$ Thus $\star$ is an absorptive element.

${ }^{11}$ We could also construct a free $\operatorname{Sem}(\mathcal{Q})$-algebra over $\mathbb{C}$, denoted $\mathbb{C}[\operatorname{Sem}(\mathcal{Q})]$, whose elements are defined to be complex-valued functions on $\operatorname{Sem}(\mathcal{Q})$ that vanish for all but a finite number of elements of $\operatorname{Sem}(\mathcal{Q})[9]$. If $u, v \in \mathbb{C}[\operatorname{Sem}(\mathcal{Q})]$, their product $u v$ is defined as $u v(h):=\sum_{f \& g=h} u(f) v(g)$. In the special case where the category $\mathcal{Q}$ is a partially-ordered set, this reproduces the incidence algebra used by Raptis and Zapatrin in their work on discretising space-time topology [4] [10] [11].
} 
on $Q$ by $(\hat{U}(g) \psi)(q):=\psi(g q)$, and this can be used as a starting point for discussing the quantum theory of a system whose configuation space is $Q$ (for more details see Section 4.1).

As anticipated in Section 1, in the category case, a crucial idea is that the arrows in a category can be thought of as 'transforming' one object into another, which suggests that perhaps $\operatorname{Hom}(\mathcal{Q})$ can play the role of the group $G$ above. Thus, if $f$ is an arrow such that $f: A \rightarrow B$, we define $\tau_{f}(A):=B$. However, this leaves open the question of how to define $\tau_{f}(A)$ when $A \neq \operatorname{Dom} f$. The simplest way (although not the one we shall ultimately adopt) is to augment the set $\operatorname{Ob}(\mathcal{Q})$ with an additional element, denoted \#, and then to define the action of an arrow $f$ on the augmented set $\mathrm{Ob}(\mathcal{Q})_{+}$by

$$
\tau_{f}(A):= \begin{cases}\operatorname{Ran} f & \text { if } \operatorname{Dom} f=A \\ \# & \text { otherwise }\end{cases}
$$

and $\tau_{f}(\#):=\#$. This can be extended to an action of the $\operatorname{semigroup} \operatorname{Sem}(\mathcal{Q})$ by defining $\tau_{\star}(A):=\#$ for all $A \in \mathrm{Ob}(\mathcal{Q})_{+}$. It is easy to check that, for all $f, g \in \operatorname{Sem}(\mathcal{Q})$,

$$
\tau_{f} \circ \tau_{g}=\tau_{f \& g} .
$$

Thus, by these means, we have defined a left action of the $\operatorname{semigroup} \operatorname{Sem}(\mathcal{Q})$ on the extended set of objects $\mathrm{Ob}(\mathcal{Q})_{+}$.

This action can be used to give a first attempt at a quantum theory on $\mathcal{Q}$. The simplest scheme is to choose state vectors to be complex-valued functions on $\mathrm{Ob}(\mathcal{Q})_{+}$and then to define operators $\hat{d}(f)$ by

$$
(\hat{d}(f) \psi)(A):=\psi\left(\tau_{f}(A)\right)
$$

for all $f \in \operatorname{Sem}(\mathcal{Q})$ and $A \in \operatorname{Ob}(\mathcal{Q})_{+}$. Then, if $f, g \in \operatorname{Sem}(\mathcal{Q})$,

$$
\begin{aligned}
(\hat{d}(g) \hat{d}(f) \psi)(A) & =(\hat{d}(f) \psi)\left(\tau_{g}(A)\right)=\psi\left(\tau_{f}\left(\tau_{g}(A)\right)\right) \\
& =\psi\left(\tau_{f \& g}(A)\right)=(\hat{d}(f \& g) \psi)(A)
\end{aligned}
$$

and so

$$
\hat{d}(g) \hat{d}(f)=\hat{d}(f \& g)
$$

which is an (anti)-representation of the semigroup $\operatorname{Sem}(\mathcal{Q})$.

One implication of Eq. (14) is that if $f \in \operatorname{Hom}(\mathcal{Q})$ but Dom $f \neq A$, then $(\hat{d}(f) \psi)(A)=\psi(\#)$, and also $(\hat{d}(\star) \psi)(A)=\psi(\#)$ for all $A \in \mathrm{Ob}(\mathcal{Q})_{+}$. In fact, nothing of significance is lost if we forget the extra element \# in 
$\mathrm{Ob}(\mathcal{Q})_{+}$in the sense that we define the state vectors to be functions on $\mathrm{Ob}(\mathcal{Q})$ only (which is equivalent to setting $\psi(\#)=0$ ): i.e., we define for $f \in \operatorname{Hom}(\mathcal{Q})$,

$$
(\hat{d}(f) \psi)(A):= \begin{cases}\psi(\operatorname{Ran} f) & \text { if Dom } f=A \\ 0 & \text { otherwise }\end{cases}
$$

and with $(\hat{d}(\star) \psi)(A):=0$ for all $A \in \mathrm{Ob}(\mathcal{Q})$. This scheme can be extended to include quantising 'configuration' variables - i.e., real-valued functions $\beta$ on $\mathrm{Ob}(\mathcal{Q})$ - by defining

$$
(\hat{\beta} \psi)(A):=\beta(A) \psi(A)
$$

for any $\beta: \mathrm{Ob}(\mathcal{Q}) \rightarrow \mathbb{R}$.

By these means we obtain a simple quantum model. But, several significant problems can be seen already. For example, no inner product has been specified on the state functions; we shall discuss this question shortly. However, the main problem is that this representation of $\operatorname{Sem}(\mathcal{Q})$ fails to separate arrows with the same domain and range. This is because if $f$ and $g$ are two such arrows then the action of $\operatorname{Sem}(\mathcal{Q})$ on $\operatorname{Ob}(\mathcal{Q})+$ in Eq. (12) is such that $\tau_{f}(A)=\tau_{g}(A)$ for all $A \in \mathrm{Ob}(\mathcal{Q})_{+}$.

This problem could be addressed using similar methods to those adopted later in the context of arrow fields. However, we will not follow this path here since, anyway, the definition Eq. (14), or Eq. (17), of the operator $\hat{d}(f)$ has some peculiar features. In particular, $\hat{d}(f)$ annihilates any function whose support lies in the complement of the singleton set $\{$ Dom $f\}$, which is rather draconian. It seems more natural to define an operator

$$
(\hat{a}(f) \psi)(A):= \begin{cases}\psi(\operatorname{Ran} f) & \text { if Dom } f=A \\ \psi(A) & \text { otherwise }\end{cases}
$$

which leaves the values of $\psi$ unchanged except on the object Dom $f$ on which the arrow $f$ naturally acts. This would correspond to an action of $\operatorname{Hom}(\mathcal{Q})$ on $\operatorname{Ob}(\mathcal{Q})$

$$
\tau_{f}^{\prime}(A):= \begin{cases}\operatorname{Ran} f & \text { if Dom } f=A \\ A & \text { otherwise }\end{cases}
$$

in contrast to Eq. (12).

However, the operators defined by Eq. (19) do not combine into themselves. For example, let $f: A \rightarrow B$ and $g: C \rightarrow D$ be arrows with $C \neq A$ 
and $A \neq D$. Then

$$
\begin{aligned}
& (\hat{a}(g) \hat{a}(f) \psi)(C)=(\hat{a}(f) \psi)(D)=\psi(D) \\
& (\hat{a}(g) \hat{a}(f) \psi)(A)=(\hat{a}(f) \psi)(A)=\psi(B) \\
& (\hat{a}(g) \hat{a}(f) \psi)(E)=\psi(E) \text { for all } E \text { not equal to } C \text { or } A .
\end{aligned}
$$

But this is not of the form $\hat{a}(h) \psi$ for any arrow $h$.

The problem lies in the definition Eq. (8) of the combination law on $\operatorname{Sem}(\mathcal{Q})$ whereby the partial semigroup $\operatorname{Hom}(\mathcal{Q})$ is transformed into a full semigroup. The introduction of the additional element $\star$ is a rather crude device, and distinctly ad hoc. As we shall now see, there is a far more elegant way of associating a full semigroup with the set of $\operatorname{arrows} \operatorname{Hom}(\mathcal{Q})$, and it is within this framework that the quantisation scheme will be developed further.

\section{The Monoid of Arrow Fields}

\subsection{The Idea of an Arrow Field}

The constructions used above are very 'local' in object space. For example, when $\tau_{f}$ acts on $\mathrm{Ob}(\mathcal{Q})_{+}$, the only object that is affected is Dom $f$ : the rest are mapped to the 'dustbin' element $\star$ that is appended to $\mathrm{Ob}(\mathcal{Q})$. On the other hand, in the motivating case of a group $G$ that acts on a manifold $Q$, each element $g \in G$ acts on every element $q$ of $Q$ (of course, this includes the case where $g$ leaves $q$ fixed), without the need to append anything to $Q$. This suggests that it would be profitable to drop the use of $\star$, and to seek an alternative structure that better resembles the typical action of a group on a manifold.

We shall do this by choosing for each object $A$ an arrow whose domain is $A$ (this could be the identity arrow $\operatorname{id}_{A}: A \rightarrow A$ ), and then act on $A$ with it. Thus we consider maps $X: \operatorname{Ob}(\mathcal{Q}) \rightarrow \operatorname{Hom}(\mathcal{Q})$ such that, for each $A \in \mathrm{Ob}(\mathcal{Q})$, the domain ${ }^{12}$ of $X(A)$ is $A$; thus $X(A): A \rightarrow B$ for some $B \in \mathrm{Ob}(\mathcal{Q})$. Such a map will be called an out-arrow field, or just an arrow field $^{13}$, on $\mathcal{Q}$.

\footnotetext{
${ }^{12}$ The map $X$ can be viewed as a cross-section of a bundle on $\operatorname{Ob}(\mathcal{Q})$ whose fibre over $A \in \mathrm{Ob}(\mathcal{Q})$ is the set of all arrows whose domain is $A$.

${ }^{13}$ Similarly, an in-arrow field is a map $Y: \operatorname{Ob}(\mathcal{Q}) \rightarrow \operatorname{Hom}(\mathcal{Q})$ such that, for each $A \in \mathrm{Ob}(\mathcal{Q})$, the range of the arrow $Y(A)$ is $A$. Only out-arrow fields will be used in what follows.
} 
For our purposes, the key property of arrow fields is that they form a full monoid without needing to append any additional elements. More precisely, if $X_{1}$ and $X_{2}$ are arrow fields, we construct an arrow field $X_{2} \& X_{1}$ by defining, for all $A \in \mathrm{Ob}(\mathcal{Q})$, the arrow $\left(X_{2} \& X_{1}\right)(A)$ to be the composition of the arrow $X_{1}(A)$ with the arrow obtained by evaluating $X_{2}$ on the range of $X_{1}(A)$ :

$$
\left(X_{2} \& X_{1}\right)(A):=X_{2}\left(\operatorname{Ran} X_{1}(A)\right) \circ X_{1}(A)
$$

Put more simply, if $X_{1}(A): A \rightarrow B$, then

$$
\left(X_{2} \& X_{1}\right)(A):=X_{2}(B) \circ X_{1}(A)
$$

as summarised in the diagram $A \stackrel{X_{1}(A)}{\longrightarrow} B \stackrel{X_{2}(B)}{\longrightarrow} C$.

To prove associativity, it is helpful to use the diagram

$$
A \stackrel{X_{1}(A)}{\longrightarrow} B \stackrel{X_{2}(B)}{\longrightarrow} C \stackrel{X_{3}(C)}{\longrightarrow} D .
$$

Then $\left[X_{3} \&\left(X_{2} \& X_{1}\right)\right](A)$ is the arrow from $A$ to $D$ given by

$$
\begin{aligned}
{\left[X_{3} \&\left(X_{2} \& X_{1}\right)\right](A) } & =X_{3}\left[\operatorname{Ran}\left(X_{2} \& X_{1}\right)(A)\right] \circ\left(X_{2} \& X_{1}\right)(A) \\
& =X_{3}(C) \circ\left(X_{2} \& X_{1}\right)(A) \\
& =X_{3}(C) \circ\left[X_{2}(B) \circ X_{1}(A)\right]
\end{aligned}
$$

for all $A \in \mathrm{Ob}(\mathcal{Q})$. On the other hand

$$
\begin{aligned}
{\left[\left(X_{3} \& X_{2}\right) \& X_{1}\right](A) } & =\left(X_{3} \& X_{2}\right)(B) \circ X_{1}(A) \\
& =\left[X_{3}(C) \circ X_{2}(B)\right] \circ X_{1}(A)
\end{aligned}
$$

for all $A \in \mathrm{Ob}(\mathcal{Q})$. Thus the arrow-field associativity property, $X_{3} \&\left(X_{2} \& X_{1}\right)=$ $\left(X_{3} \& X_{2}\right) \& X_{1}$, follows from the associativity of arrow composition in the category $\mathcal{Q}$.

There is also a unit element for the \&-algebraic structure. This is the arrow field $\iota$ defined by

$$
\iota(A):=\operatorname{id}_{A}
$$

for all $A \in \operatorname{Ob}(\mathcal{Q})$. Thus the set of all arrow fields on $\mathcal{Q}$ is a full monoid. We will denote it $\operatorname{AF}(\mathcal{Q}){ }^{14}$

\footnotetext{
${ }^{14}$ If desired, an 'incidence algebra' $\mathbb{C}[\operatorname{AF}(\mathcal{Q})]$ can be associated with the monoid $\operatorname{AF}(\mathcal{Q})$ in the same way that $\mathbb{C}[\operatorname{Sem}(\mathcal{Q})]$ is generated by $\operatorname{Sem}(\mathcal{Q})$.
} 


\subsection{The Action of $\operatorname{AF}(\mathcal{Q})$ on $\operatorname{Ob}(\mathcal{Q})$}

The definition of an arrow field is such that, for each object $A, X(A)$ is an arrow whose domain is $A$. Thus we can define an action $\ell$ of the monoid $\operatorname{AF}(\mathcal{Q})$ on the set $\mathrm{Ob}(\mathcal{Q})$ by letting $X \in \mathrm{AF}(\mathcal{Q})$ transform $A \in \mathrm{Ob}(\mathcal{Q})$ into the range of the arrow $X(A)$ :

$$
\ell_{X}(A):=\operatorname{Ran} X(A) .
$$

In other words, if $X(A): A \rightarrow B$ then $\ell_{X}(A):=B$.

This defines a genuine monoid action of $\operatorname{AF}(\mathcal{Q})$ on $\mathrm{Ob}(\mathcal{Q})$, since, for all $A \in \mathrm{Ob}(\mathcal{Q})$,

$$
\begin{aligned}
\ell_{X_{2} \& X_{1}}(A) & :=\operatorname{Ran}\left[\left(X_{2} \& X_{1}\right)(A)\right]=\operatorname{Ran}\left[X_{2}\left(\operatorname{Ran} X_{1}(A)\right) \circ X_{1}(A)\right] \\
& =\operatorname{Ran}\left[X_{2}\left(\operatorname{Ran} X_{1}(A)\right)\right]
\end{aligned}
$$

whereas

$$
\ell_{X_{2}}\left(\ell_{X_{1}}(A)\right)=\ell_{X_{2}}\left(\operatorname{Ran} X_{1}(A)\right)=\operatorname{Ran}\left[X_{2}\left(\operatorname{Ran} X_{1}(A)\right)\right]
$$

and hence, for all $X_{1}, X_{2} \in \operatorname{AF}(\mathcal{Q})$,

$$
\ell_{X_{2}} \circ \ell_{X_{1}}=\ell_{X_{2} \& X_{1}}
$$

as required.

Note that a significant difference between this action and that of a Lie group $G$ on a manifold $Q$ is that the same group element acts at each point $q \in Q$, whereas, in the arrow-field action, it is not $X$ as a whole, but rather the arrow $X(A)$ which acts at $A \in \mathrm{Ob}(\mathcal{Q})$, and this arrow is arbitrary for each $A$. Thus the arrow-field transformations are more like the action on $Q$ of the full diffeomorphism group $\operatorname{Diff}(Q)$ than that of the finite-dimensional subgroup $G$. If one wanted to emulate the familiar group case more closely it would be necessary to relate the arrows at different objects in some way. For most categories $\mathcal{Q}$ there is no obvious way of doing this since the different objects in the category are frequently very different from each other. ${ }^{15}$ There are some specific examples of 'constant' arrow fields in [7].

\subsection{The Special Arrow-Fields $X_{f}$}

An arrow-field $X$ assigns an arbitrary arrow $X(A)$ to each object $A$ subject only to the requirement that $\operatorname{Dom} X(A)=A$. However, a particularly

\footnotetext{
${ }^{15}$ One exception is when a manifold $Q \simeq G / H$ is regarded as a category in the way discussed later in Section 4.3.1. In this case, we can define the special arrow fields $X^{g}$, $g \in G$, as $X^{g}(q): q \rightarrow g q$ for all $q \in Q$.
} 
simple choice of $X$ is when all but one of the arrows $X(A), A \in \mathrm{Ob}(\mathcal{Q})$, is the identity $\operatorname{id}_{A}$. More precisely, for each arrow $f \in \operatorname{Hom}(\mathcal{Q})$, an arrow field $X_{f}$ can be defined by

$$
X_{f}(A):= \begin{cases}f & \text { if Dom } f=A \\ \operatorname{id}_{A} & \text { otherwise }\end{cases}
$$

for all $A \in \mathrm{Ob}(\mathcal{Q})$.

The action of $X_{f}$ on the $\operatorname{set} \operatorname{Ob}(\mathcal{Q})$ is

$$
\ell_{X_{f}}(A)= \begin{cases}\operatorname{Ran} f & \text { if Dom } f=A \\ A & \text { otherwise }\end{cases}
$$

which should be contrasted with the definition of $\tau_{f}$ in Eq. (12). The transformation Eq. (35) was anticipated in Eq. (20).

A natural extension is to pick any finite set of elements $f_{1}, f_{2}, \ldots, f_{n} \in$ $\operatorname{Hom}(\mathcal{Q})$, each of which has a different domain from the others. We can then define the arrow-field

$$
X_{f_{1}, f_{2}, \ldots, f_{n}}(A):= \begin{cases}f_{i} & \text { if Dom } f_{i}=A, i=1,2, \ldots, n \\ \operatorname{id}_{A} & \text { otherwise. }\end{cases}
$$

Arrow fields of this type have finite support, where the support of an arrow field is defined to be the set of all objects $A \in \mathrm{Ob}(\mathcal{Q})$ such that $X(A) \neq \operatorname{id}_{A}$.

The collection of all arrow fields of finite support is a submonoid of $\operatorname{AF}(\mathcal{Q})$, and is likely to play an important role in a deeper analysis of the quantum theory. From a mathematical perspective, its role could perhaps be compared with that of the group of gauge transformations of compact support in a normal gauge theory (although it must be emphasised that the physical significance of $\operatorname{AF}(\mathcal{Q})$ is not the same as that of a standard gauge group).

One might anticipate that the set of arrow fields of finite support can be constructed by taking the \& product of arrow fields of the type $X_{f}$. This is true, but the order of the elements in the product is important. For example, consider a pair of arrows $f: A \rightarrow B$ and $g: C \rightarrow D$ with $A \neq C$. Then, if $B \neq C$,

$$
X_{f} \& X_{g}=X_{g} \& X_{f}=X_{f, g}
$$

On the other hand, if $B=C$, so that we have the chain $A \stackrel{f}{\longrightarrow} B \stackrel{g}{\longrightarrow} D$, and if $A \neq B$, then $X_{f} \& X_{g}=X_{f, g}$ but $X_{g} \& X_{f}=X_{g \circ f, g} \neq X_{f, g}$. 
Finally, note that if $\operatorname{Dom} f \neq \operatorname{Ran} f$ then

$$
X_{f} \& X_{f}=X_{f}
$$

so these are idempotent elements of the monoid $\operatorname{AF}(\mathcal{Q})$. On the other hand, if Dom $f=\operatorname{Ran} f$ then $X_{f} \& X_{f}=X_{f \circ f}$.

\section{Arrow-Field Quantum Theory}

\subsection{Quantisation on a Manifold $Q$}

To motivate what follows, consider first a classical system whose configuration space is a manifold $Q$ on which there is a transitive left action by a Lie group $G$ with $Q \simeq G / H$, where $H$ is a closed subgroup of $G$. Thus, to each $g \in G$ there is a diffeomorphism $\tau_{g}: Q \rightarrow Q$ with

$$
\tau_{g_{2}} \circ \tau_{g_{1}}=\tau_{g_{2} g_{1}}
$$

for all $g_{1}, g_{2} \in G$.

The classical state space is the cotangent bundle $T^{*} Q$, and the quantisation scheme advocated in [6] involves finding the smallest finite-dimensional group of symplectic transformations that acts transitively on $T^{*} Q$. This is a semi-direct product $G \times{ }_{\tau} W$ (the ' $\tau$ ' denotes the action of $G$ on $Q$ ) where $W$ (a finite-dimensional, linear subspace of $C^{\infty}(Q)$ ) is the dual of the smallest vector space that carries a linear representation of $G$ with a $G$-orbit that is equivariantly diffeomorphic to $G / H$. Induced representation theory [12] shows that the main class of unitary irreducible representations of $G \times_{\tau} W$ is given by vector bundles over this orbit, in which the vector-space fibre carries an irreducible representation of $H .^{16}$

Note that $G \times_{\tau} W$ is a finite-dimensional subgroup of the (infinitedimensional) group of symplectic transformations, $\operatorname{Diff}(Q) \times{ }_{d} C^{\infty}(Q)$, of $T^{*} Q$ (where $d$ denotes the action on $Q$ of the diffeomorphism group, $\operatorname{Diff}(Q)$, of $Q)$. Many of the representations of $G \times{ }_{\tau} W$ extend to the group $G \times{ }_{\tau} C^{\infty}(Q)$, and some of these extend to $\operatorname{Diff}(Q) \times{ }_{d} C^{\infty}(Q)$. However, the general representation theory of the infinite-dimensional group $\operatorname{Diff}(Q) \times{ }_{d} C^{\infty}(Q)$ is far more complicated, and incomplete, than that of its finite-dimensional subgroup $G \times{ }_{\tau} W$. Unfortunately, if the manifold $Q$ is not a homogeneous space $G / H$, then usually one has to fall back on using $\operatorname{Diff}(Q) \times{ }_{d} C^{\infty}(Q)$.

\footnotetext{
${ }^{16}$ There may also be 'atypical' representations in which the vector bundle is over an orbit that is not diffeomorphic to $Q$.
} 
It would be good to develop a complete analogy of this scheme for a general small category $\mathcal{Q}$. However, this involves finding an appropriate analogue of symplectic geometry, which is not obvious. Here, we will adopt a more heuristic approach in which we start by thinking of state vectors as being merely complex-valued functions on $\mathrm{Ob}(\mathcal{Q})$, and then see where this leads in the construction of the analogue of the quantisation group $G \times{ }_{\tau} W$; or, perhaps more precisely, of the group $\operatorname{Diff}(Q) \times{ }_{d} C^{\infty}(Q)$.

In the manifold case, if there is some $G$-invariant measure $\mu$ on $Q$, then a representation of $G$ on the Hilbert space $L^{2}(Q, d \mu)$ of complex-valued functions on $Q$ can be defined by

$$
(\hat{U}(g) \psi)(q):=\psi\left(\tau_{g^{-1}}(q)\right),
$$

so that $\hat{U}\left(g_{2}\right) \hat{U}\left(g_{1}\right)=\hat{U}\left(g_{2} g_{1}\right)$ for all $g_{1}, g_{2} \in G$. If $\mu$ is invariant under the action of $G$ on $Q$ then this representation is unitary. Note that if $\hat{U}(g)$ is defined instead as

$$
(\hat{U}(g) \psi)(q):=\psi\left(\tau_{g}(q)\right)
$$

then $\hat{U}\left(g_{2}\right) \hat{U}\left(g_{1}\right)=\hat{U}\left(g_{1} g_{2}\right)$ for all $g_{1}, g_{2} \in G$, i.e., we get an anti-representation of $G$.

When $Q \simeq G / H$, the representation Eq. (40) can be used as the basis for a simple quantisation of the system. This involves defining operator representations of configuration variable functions $\beta \in C^{\infty}(Q)$, by

$$
(\hat{\beta} \psi)(q):=\beta(q) \psi(q)
$$

which can be exponentiated to give the unitary operators

$$
(\hat{V}(\beta) \psi)(q):=e^{-i \beta(q)} \psi(q) .
$$

Together, $\hat{U}(g)$ and $\hat{V}(\beta)$ satisfy the relations

$$
\begin{aligned}
\hat{U}\left(g_{1}\right) \hat{U}\left(g_{2}\right) & =\hat{U}\left(g_{1} g_{2}\right) \\
\hat{V}\left(\beta_{1}\right) \hat{V}\left(\beta_{2}\right) & =\hat{V}\left(\beta_{1}+\beta_{2}\right) \\
\hat{U}(g) \hat{V}(\beta) & =\hat{V}\left(\beta \circ \tau_{g^{-1}}\right) \hat{U}(g)
\end{aligned}
$$

where $\beta \circ \tau_{g^{-1}}(q):=\beta\left(\tau_{g^{-1}}(q)\right)$. If the definition Eq. (41) is used instead of Eq. (40), we get the relations

$$
\begin{aligned}
\hat{U}\left(g_{2}\right) \hat{U}\left(g_{1}\right) & =\hat{U}\left(g_{1} g_{2}\right) \\
\hat{V}\left(\beta_{1}\right) \hat{V}\left(\beta_{2}\right) & =\hat{V}\left(\beta_{1}+\beta_{2}\right) \\
\hat{U}(g) \hat{V}(\beta) & =\hat{V}\left(\beta \circ \tau_{g}\right) \hat{U}(g) .
\end{aligned}
$$


For this system, Eqs. (44-46) (or Eqs. (47-49)) are the analogue of the (exponentiated) canonical commutation relations of elementary wave mechanics. ${ }^{17}$ They constitute a representation of the subgroup $G \times{ }_{\tau} C^{\infty}(Q)$ of the much larger group $\operatorname{Diff}(Q) \times{ }_{d} C^{\infty}(Q)$.

\subsection{The Basic Algebra for the Quantum Theory on $\mathcal{Q}$}

Our first task is to find the analogue of Eqs. (47-49) for a system whose configuration space (or history-theory equivalent) is the set of objects $\mathrm{Ob}(\mathcal{Q})$ in a small category $\mathcal{Q}$. The key idea is to use the monoid $\operatorname{AF}(\mathcal{Q})$ as an analogue of the group of diffeomorphisms of $Q$.

We start with the simplest approach to constructing a quantum theory on $\mathcal{Q}$, which is to take the state vectors to be complex-valued functions on $\operatorname{Ob}(\mathcal{Q})$. The action of the monoid $\operatorname{AF}(\mathcal{Q})$ on such functions is (writing $\ell_{X}(A)$ as $\ell_{X} A$ for typographical clarity)

$$
(\hat{a}(X) \psi)(A):=\psi\left(\ell_{X} A\right)=\psi[\operatorname{Ran} X(A)]
$$

which is like the earlier definition Eq. (14) except that there is no need to augment the set $\mathrm{Ob}(\mathcal{Q})$ with the additional element \#.

We have

$$
\begin{aligned}
\left(\hat{a}\left(X_{2}\right) \hat{a}\left(X_{1}\right) \psi\right)(A) & =\left(\hat{a}\left(X_{1}\right) \psi\right)\left(\ell_{X_{2}} A\right)=\psi\left(\ell_{X_{1}}\left(\ell_{X_{2}} A\right)\right)=\psi\left(\ell_{X_{1} \& X_{2}} A\right) \\
& =\left(\hat{a}\left(X_{1} \& X_{2}\right) \psi\right)(A)
\end{aligned}
$$

where Eq. (33) has been used. Thus we have an anti-representation of the monoid $\operatorname{AF}(\mathcal{Q})$ :

$$
\hat{a}\left(X_{2}\right) \hat{a}\left(X_{1}\right)=\hat{a}\left(X_{1} \& X_{2}\right)
$$

for all $X_{1}, X_{2} \in \operatorname{AF}(\mathcal{Q})$.

If Eq. (50) is applied to the special arrow fields $X_{f}$ in Eq. (34) then, defining $\hat{a}(f):=\hat{a}\left(X_{f}\right)$, we get

$$
(\hat{a}(f) \psi)(A)= \begin{cases}\psi(\operatorname{Ran} f) & \text { if } \operatorname{Dom} f=A \\ \psi(A) & \text { otherwise }\end{cases}
$$

\footnotetext{
${ }^{17}$ To be more precise, this is so when the functions $\beta: Q \rightarrow \mathbb{R}$ are restricted to belong to the finite-dimensional subspace $W \subset C^{\infty}(Q)$ mentioned earlier. However, a representation of $G \times{ }_{\tau} W$ on sections of vector bundles over $Q$ can be extended to include all $C^{\infty}$ functions on $Q$ (modulo the usual subtleties with operator domains).
} 
as anticipated in Eq. (19). The 'closure' problem that arose earlier in the context of Eqs. (21-23) no longer applies since the monoid product of two arrow fields $X_{f}, X_{g}$ is itself an arrow field (albeit, possibly not of the form $X_{h}$ for any $h \in \operatorname{Hom}(\mathcal{Q})$ ).

One might wonder what the adjoint of an operator $\hat{a}(X)$ looks like, but this cannot be answered before putting an inner product on the state functions, which of course is essential anyway to the physical interpretation of the theory. However, there is no obvious way of doing this in general. If $\mathrm{Ob}(\mathcal{Q})$ is a finite, or countably infinite, set we can define

$$
\langle\phi \mid \psi\rangle:=\sum_{A \in \mathrm{Ob}(\mathcal{Q})} \phi(A)^{*} \psi(A)
$$

although it would be nice to have some specific physical, or mathematical, reason for choosing this particular inner product.

More generally, we need to explore the construction of 'appropriate' measures $\mu$ on $\operatorname{Ob}(\mathcal{Q})$ so that we can define

$$
\langle\phi \mid \psi\rangle:=\int_{\mathrm{Ob}(\mathcal{Q})} d \mu(A) \phi^{*}(A) \psi(A) .
$$

The first step is to find fields of measurable sets on $\mathrm{Ob}(\mathcal{Q})$, and the easiest way to do this is if there is a topology on $\mathrm{Ob}(\mathcal{Q})$. For example, in the special case where $\mathcal{Q}$ is a poset $P$ there are the order topologies on $P$ (i.e. generated by the upper or lower sets of $P$ ) and there are probably analogues of these on a general small category. However, this is a complicated issue, and is deferred to a later paper. For the purposes of the present paper it will be assumed that $\mathrm{Ob}(\mathcal{Q})$ is finite or countable, so that the simple inner product Eq. (54) can be used. This is reasonable since many of the physically interesting examples do have a countable collection of objects.

The next step in the construction of our 'category quantisation monoid' is to represent the space of real-valued functions on $\mathrm{Ob}(\mathcal{Q})$ (the 'configuration variables') by

$$
(\hat{\beta} \psi)(A):=\beta(A) \psi(A)
$$

as in Eq. (18).

The crucial task now is to extract an algebra from the operators $\hat{a}(X)$ and $\hat{\beta}$. To this end, we first compute

$$
(\hat{a}(X) \hat{\beta} \psi)(A)=(\hat{\beta} \psi)\left(\ell_{X} A\right)=\beta\left(\ell_{X} A\right) \psi\left(\ell_{X} A\right)
$$


while

$$
(\hat{\beta} \hat{a}(X) \psi)(A)=\beta(A)(\hat{a}(X) \psi)(A)=\beta(A) \psi\left(\ell_{X} A\right)
$$

which implies that

$$
\left(\widehat{\beta \circ \ell_{X}} \hat{a}(X) \psi\right)(A)=\beta\left(\ell_{X} A\right) \psi\left(\ell_{X} A\right)
$$

where $\widehat{\beta \circ \ell_{X}}$ is defined by $\left(\widehat{\beta \circ \ell_{X}} \psi\right)(A):=\beta\left(\ell_{X} A\right) \psi(A)$ for all $A \in \operatorname{Ob}(\mathcal{Q})$. From Eq. (57) and Eq. (59) we obtain the relation

$$
\hat{a}(X) \hat{\beta}=\widehat{\beta \circ \ell_{X}} \hat{a}(X)
$$

for all $X \in \operatorname{AF}(\mathcal{Q})$ and functions $\beta: \mathrm{Ob}(\mathcal{Q}) \rightarrow \mathbb{R}$.

Next we introduce the unitary operator $\hat{V}(\beta):=\exp -i \hat{\beta}$ which satisfies $\hat{V}\left(\beta_{1}\right) \hat{V}\left(\beta_{2}\right)=\hat{V}\left(\beta_{1}+\beta_{2}\right)$ for all functions $\beta_{1}$ and $\beta_{2}$. Finally, putting these relations together, we get

$$
\begin{aligned}
\hat{a}\left(X_{2}\right) \hat{a}\left(X_{1}\right) & =\hat{a}\left(X_{1} \& X_{2}\right) \\
\hat{V}\left(\beta_{1}\right) \hat{V}\left(\beta_{2}\right) & =\hat{V}\left(\beta_{1}+\beta_{2}\right) \\
\hat{a}(X) \hat{V}(\beta) & =\hat{V}\left(\beta \circ \ell_{X}\right) \hat{a}(X)
\end{aligned}
$$

which should be viewed as the category analogue of Eqs. (47-49). In the manifold case of Eqs. (47-49) we have a representation of the group $G \times \times_{\tau}$ $C^{\infty}(Q)$. In the category case of Eqs. (61-63) we have a representation of the semi-direct product $\operatorname{AF}(\mathcal{Q}) \times_{\ell} F(\mathrm{Ob}(\mathcal{Q}), \mathbb{R})$ of the monoid $\operatorname{AF}(\mathcal{Q})$ with the vector space $F(\mathrm{Ob}(\mathcal{Q}), \mathbb{R})$ of all real-valued functions on $\mathrm{Ob}(\mathcal{Q})$. In what follows, $\operatorname{AF}(\mathcal{Q}) \times_{\ell} F(\mathrm{Ob}(\mathcal{Q}), \mathbb{R})$ will be called the 'category quantisation monoid'.

Note, however, that in the manifold case, the functions $\beta: Q \rightarrow \mathbb{R}$ are not totally arbitrary. At the very least, they are required to be measurable with respect to the natural $\sigma$-algebra of sets associated with the topology on $Q$; and one may well wish to restrict them to be $C^{\infty}$. However, no analogous structure has yet been placed on $\operatorname{Ob}(\mathcal{Q})$, and therefore, as things stand, the only option is to include all real-valued functions on $\mathrm{Ob}(\mathcal{Q})$.

Modulo this caveat, the central idea of the proposed quantum scheme is that the possible quantum theories on $\mathcal{Q}$ are given by the different faithful, irreducible representations of the category quantisation monoid $\operatorname{AF}(\mathcal{Q}) \times_{\ell}$ $F(\mathrm{Ob}(\mathcal{Q}), \mathbb{R})$. Each such representation will satisfy the relations in Eqs. (6163). However, some important questions arise when comparing these with the analogous relations Eqs. (47-49) for the case where the configuration 
space is a manifold $Q \simeq G / H$. For example, the group $G$ acts transitively on $Q$, and this is an important requirement in proving the irreducibility of the representation of the quantisation group $G \times{ }_{\tau} C^{\infty}(Q)$. It is necessary to explore the analogue for the action of the monoid $\operatorname{AF}(\mathcal{Q})$ on $\operatorname{Ob}(\mathcal{Q})$. This issue will be discussed briefly in Section 4.7, and is examined in more detail in a later paper.

Note that the operators $\hat{U}(g), g \in G$, in Eqs. (47-49) are unitary, but this will not be the case for the operators $\hat{a}(X)$, irrespective of the choice of the measure $\mu$ on $\mathrm{Ob}(\mathcal{Q})$. Indeed, although it is natural to view an arrow as the analogue of momentum - in the sense that it transforms one object to another - objects in a category of structured sets are typically very different from each other and, in this sense, $\hat{a}(X)$ is a type of creation or annihilation operator. In Section 4.4 we shall see how this works in specific examples.

\subsection{Introducing a Multiplier}

\subsubsection{The Basic Ideas}

In the context of arrow fields, we shall say that two arrows $f, g$ are separated in the quantum theory if $\hat{a}\left(X_{f}\right) \neq \hat{a}\left(X_{g}\right)$. In this respect, the representation of the category quantisation monoid constructed above is inadequate since it fails to separate arrows that have the same domain and range: indeed, if $f, g$, are any two such arrows then $\hat{a}\left(X_{f}\right) \psi=\hat{a}\left(X_{g}\right) \psi$ for all states $\psi$. In particular, it cannot represent any of the internal structure of the objects in the category as reflected in the sets $\operatorname{Hom}(A, A), A \in \mathrm{Ob}(\mathcal{Q})$. To get such a separation, the crucial step is to refine the quantum scheme by letting the state functions $\psi$ take their values in a Hilbert space $\mathcal{K}$ that is larger than $\mathbb{C}$.

To motivate what follows, we note first that a system with a configuration manifold $Q \simeq G / H$, where $G$ is a Lie group, can be viewed as a special example of this categorial structure. Specifically: let $\mathcal{Q}$ be the category whose objects are the points in $Q$, and whose arrows from $q_{1} \in Q$ to $q_{2} \in Q$ are defined to be the group elements $g \in G$ such that $q_{2}=g q_{1}$, where $g q$ denotes the point in $Q$ obtained by acting on $q \in Q$ with $g \in G$ (i.e., $\left.g q:=\tau_{g}(q)\right)$. Thus

$$
\operatorname{Hom}\left(q_{1}, q_{2}\right):=\left\{g \in G \mid q_{2}=g q_{1}\right\} .
$$

Composition of group elements regarded as arrows ${ }^{18}$ is just the group prod-

\footnotetext{
${ }^{18}$ In this example, an arrow field is defined by a function $X: Q \rightarrow G$. Since we are dealing with manifolds, it would be natural to require this function to be smooth.
} 
uct. Thus if $g_{1}: q_{1} \rightarrow q_{2}$ (i.e., $\left.q_{2}=g_{1} q_{1}\right)$ and $g_{2}: q_{2} \rightarrow q_{3}$ (i.e., $q_{3}=g_{2} q_{2}$ ), then we define $g_{2} \circ g_{1}: q_{1} \rightarrow q_{3}$ as $g_{2} g_{1}: q_{1} \rightarrow q_{3}$. The associativity of the composition of arrows follows from the associativity of the group product ${ }^{19}$. In particular, Eq. (64) gives

$$
\operatorname{Hom}(q, q)=\{g \in G \mid q=g q\}=G_{q}
$$

where $G_{q}$ denotes the 'little group' (or stability group) of the $G$-action at the point $q \in Q$.

Now suppose that $g_{1}, g_{2}$ be arrows with the same domain and range, so that $g_{1}: q_{1} \rightarrow q_{2}$ and $g_{2}: q_{1} \rightarrow q_{2}$ for some $q_{1}, q_{2} \in Q$. Thus $q_{2}=g_{1} q_{1}$ and $q_{2}=g_{2} q_{1}$, so that $q_{1}=g_{1}^{-1} g_{2} q_{1}$, and hence $g_{1}^{-1} g_{2}$ belongs to the stability group $G_{q_{1}}$, which is isomorphic to $H$. If we denote $h:=g_{1}^{-1} g_{2} \in G_{q_{1}}$, then $g_{2}=g_{1} h$; or, in arrow language, $g_{2}=g_{1} \circ h$ where $h \in \operatorname{Hom}\left(q_{1}, q_{1}\right)$. Thus, to separate the arrows $g_{1}$ and $g_{2}$ (with domain $q_{1}$ ) it suffices that $G_{q_{1}} \simeq H$ be represented faithfully on $\mathcal{K}$. This is because, if $R(g)$ denotes the representation of $g \in G$, then $R\left(g_{2}\right)=R\left(g_{1}\right) R(h)$, and hence $R\left(g_{2}\right) R\left(g_{1}\right)^{-1}=R(h)$, which, for $h \neq e$ (the identity element in $G_{q_{1}}$ ), will not equal the unit operator if the representation of $H$ is faithful.

However, in a general small category $\mathcal{Q}$, if $f_{1}, f_{2} \in \operatorname{Hom}(A, B)$ this does not imply the existence of an arrow $\alpha: A \rightarrow A$ such that $f_{2}=f_{1} \circ \alpha$, or an arrow $\beta: B \rightarrow B$ such that $f_{2}=\beta \circ f_{1}$, or even a pair of arrows $\alpha: A \rightarrow A$, $\beta: B \rightarrow B$ such that $f_{2}=\beta \circ f_{1} \circ \alpha$. Nevertheless, the arrows that need to be distinguished certainly include those in the $\operatorname{sets} \operatorname{Hom}(A, A), A \in \mathrm{Ob}(\mathcal{Q})$, and these are generally object-dependent. This suggest strongly that, in general, $\mathcal{K}$ must be object dependent.

In the manifold case when $G$ acts on $Q$, the standard procedure [13] for finding group representations using $\mathcal{K}$-valued functions requires the introduction of a family of linear maps $m(g, q): \mathcal{K} \rightarrow \mathcal{K}, g \in G, q \in Q$, (a so-called 'multiplier') and then defining $(\hat{U}(g) \psi)(q)=m(g, q) \psi(g q)$. Therefore, in the case of a general small category, we are led to consider a family of Hilbert spaces $\mathcal{K}[A], A \in \mathrm{Ob}(\mathcal{Q})$, with a multiplier, $m(X, A)$, that is a linear map from $\mathcal{K}\left[\ell_{X} A\right]$ to $\mathcal{K}[A]$.

To summarise: we take a bundle of Hilbert spaces $\bigcup_{A \in \mathrm{Ob}(\mathcal{Q})} \mathcal{K}[A]$ over $\mathrm{Ob}(\mathcal{Q})$, whose cross-sections are to be identified as the quantum states. For

\footnotetext{
${ }^{19}$ This is a generalisation of the well-known fact that a group can be regarded as a category with a single object, and whose arrows are the group elements. In fact, since each arrow is invertible, the category associated with $Q$ is a groupoid.
} 
a specific measure $\mu$ on $\mathrm{Ob}(\mathcal{Q})$, the inner product is

$$
\langle\phi \mid \psi\rangle:=\int_{\mathrm{Ob}(\mathcal{Q})} d \mu(A)\langle\phi(A), \psi(A)\rangle_{\mathcal{K}[A]}
$$

where $\langle\phi(A), \psi(A)\rangle_{\mathcal{K}[A]}$ denotes the inner product in the Hilbert space $\mathcal{K}[A]$. The arrow-field operator is defined as

$$
(\hat{a}(X) \psi)(A):=m(X, A) \psi\left(\ell_{X} A\right)
$$

where $\ell_{X}(A):=\operatorname{Ran} X(A)$, and

$$
m(X, A): \mathcal{K}\left[\ell_{X} A\right] \rightarrow \mathcal{K}[A]
$$

is a linear map from $\mathcal{K}\left[\ell_{X} A\right]$ to $\mathcal{K}[A]$. The configuration variables are realvalued functions $\beta$ on $\mathrm{Ob}(\mathcal{Q})$, and are represented (in exponentiated form) by the unitary operators

$$
(\hat{V}(\beta) \psi)(A):=e^{-i \beta(A)} \psi(A) .
$$

Using Eqs. (67-69), it is easy to check that Eq. (63) is satisfied, and of course Eq. (62) remains unchanged. However, to satisfy Eq. (61) certain conditions must be imposed on the multipliers. Specifically, we have

$$
(\hat{a}(Y \& X) \psi)(A)=m(Y \& X, A) \psi\left(\ell_{Y \& X} A\right)=m(Y \& X, A) \psi\left(\ell_{Y}\left(\ell_{X} A\right)\right)
$$

while

$$
\begin{aligned}
(\hat{a}(X) \hat{a}(Y) \psi)(A) & =m(X, A)(\hat{a}(Y) \psi)\left(\ell_{X} A\right) \\
& =m(X, A) m\left(Y, \ell_{X} A\right) \psi\left(\ell_{Y}\left(\ell_{X} A\right)\right) .
\end{aligned}
$$

Hence the required condition is

$$
m(Y \& X, A)=m(X, A) m\left(Y, \ell_{X} A\right)
$$

for all arrow fields $X, Y$ and all $A \in \mathrm{Ob}(\mathcal{Q})$.

Note that if $X$ and $Y$ are such that $X(A): A \rightarrow A$ and $Y(A): A \rightarrow A$, then Eq. (72) gives

$$
m(Y \& X, A)=m(X, A) m(Y, A)
$$

which corresponds to an anti-representation of the monoid $\operatorname{Hom}(A, A)$ on the Hilbert space $\mathcal{K}[A]$.

The goal in choosing the individual Hilbert spaces $\mathcal{K}[A]$ is to distinguish different arrows between the same objects. Hence, in particular, the representation Eq. (73) of the monoid $\operatorname{Hom}(A, A)$ on $\mathcal{K}[A]$ must be faithful for 
all $A \in \mathrm{Ob}(\mathcal{Q})$. Of course, this may not be sufficient to distinguish arrows between different objects.

It is possible to introduce multipliers even when the state vectors are only complex-valued functions on $\operatorname{Ob}(\mathcal{Q})$. A multiplier would then be a family of complex numbers $m(X, A), X \in \operatorname{AF}(\mathcal{Q}), A \in \mathrm{Ob}(\mathcal{Q})$, satisfying the consistency conditions in Eq. (72). However, such an addition to the simple quantum theory is unlikely to help with the problem of separating arrows with the same domain and range. For example, in a category of sets, the monoid $\operatorname{Hom}(A, A)$ is non-abelian for any set $A$ with more than one element: as such, it cannot be represented faithfully with multipliers that are complex numbers.

\subsubsection{Equivalent and Inequivalent Multipliers}

Let us now discuss briefly the question of when two multipliers give rise to unitarily equivalent representations of the category quantisation monoid. Consider a function $A \mapsto L(A)$ which to each $A \in \mathrm{Ob}(\mathcal{Q})$ associates an invertible linear operator in the Hilbert space $\mathcal{K}[A]$ (i.e., $L(A) \in G L(\mathcal{K}[A])$ ). Let $B:=\operatorname{Ran} X(A)$ and $C:=\operatorname{Ran} Y(\operatorname{Ran} X(A)$ ) (so that $X(A): A \rightarrow B$ and $Y(B): B \rightarrow C)$ and define a linear map $m_{L}(X, A): \mathcal{K}[B] \rightarrow \mathcal{K}[A]$ by

$$
m_{L}(X, A):=L(A) m(X, A) L(B)^{-1}
$$

for all $X \in \operatorname{AF}(\mathcal{Q})$ and $A \in \mathrm{Ob}(\mathcal{Q})$. Then

$$
m_{L}(Y \& X, A)=L(A) m(Y \& X, A) L^{-1}(C),
$$

whereas

$$
\begin{aligned}
m_{L}(X, A) m_{L}(Y, B) & =L(A) m(X, A) L(B)^{-1} L(B) m(Y, B) L(C)^{-1} \\
& =L(A) m(X, A) m(Y, B) L(C)^{-1}=L(A) m(Y \& X, A) L(C)^{-1} \\
& =m_{L}(Y \& X, A) .
\end{aligned}
$$

Hence Eq. (72) is satisfied, and so $m_{L}(X, A):=L(A) m(X, A) L(B)^{-1}$ is also a multiplier.

If the operators $L(A)$ are unitary for all $A \in \mathrm{Ob}(\mathcal{Q})$ (i.e., $L(A) \in$ $U(\mathcal{K}[A]))$, then the representation of the monoid $\operatorname{AF}(\mathcal{Q})$ defined by $(\hat{a}(X) \psi)(A):=$ $m_{L}(X, A) \psi\left(\ell_{X} A\right)$ is clearly unitarily equivalent to the one obtained using $m(X, A)$. This suggests that there is a family of inequivalent multipliers classified by functions $A \mapsto G L(\mathcal{K}[A]) / U(\mathcal{K}[A]) .{ }^{20}$

\footnotetext{
${ }^{20}$ Following the nomenclature used in group theory, we could say that a quantity $m$
} 


\subsubsection{The Presheaf Perspective}

As things stand ${ }^{21}$, the linear map $m(X, A): \mathcal{K}[\operatorname{Ran} X(A)] \rightarrow \mathcal{K}[A]$ could depend on the values of the arrow-field $X$ at objects $B$ other than $A$. However, such a 'non-local' property seems unnatural, and from now on we will suppose that the dependence of $m(X, A)$ on $X \in \mathrm{AF}(\mathcal{Q})$ and $A \in \mathrm{Ob}(\mathcal{Q})$ is via the arrow $X(A)$ only. Hence

$$
m(X, A)=\kappa(X(A))
$$

for some $\kappa(X(A)): \mathcal{K}[\operatorname{Ran} X(A)] \rightarrow \mathcal{K}[A]$.

The consistency conditions Eq. (72) on the multiplier become

$$
\kappa[(Y \& X)(A)]=\kappa[X(A)] \kappa[Y(B)]
$$

where $B, C \in \mathrm{Ob}(\mathcal{Q})$ are such that $X(A): A \rightarrow B$ and $Y(B): B \rightarrow C$. But $(Y \& X)(A)=Y(B) \circ X(A)$, and so Eq. (78) becomes

$$
\kappa[Y(B) \circ X(A)]=\kappa[X(A)] \kappa[Y(B)] .
$$

However, given any arrow $f \in \operatorname{Hom}(\mathcal{Q})$, there is at least one arrow field $X$ such that $X(\operatorname{Dom} f)=f$ (for example, $X_{f}$ defined in Eq. (34) has this property). Thus a multiplier $\kappa$ satisfying Eq. (78) determines linear maps $\kappa(f): \mathcal{K}[\operatorname{Ran} f] \rightarrow \mathcal{K}[\operatorname{Dom} f]$, for all $f \in \operatorname{Hom}(\mathcal{Q})$. From Eq. (79), these satisfy the conditions

$$
\kappa(g \circ f)=\kappa(f) \kappa(g)
$$

for all $f, g \in \operatorname{Hom}(\mathcal{Q})$ such that $\operatorname{Ran} f=\operatorname{Dom} g$ (so that $g \circ f$ is defined). Conversely, any family of maps $\kappa(f): \mathcal{K}[\operatorname{Ran} f] \rightarrow \mathcal{K}[\operatorname{Dom} f], f \in \operatorname{Hom}(\mathcal{Q})$, that satisfies Eq. (80), gives rise to a multiplier defined by $m(X, A):=$ $\kappa(X(A))$.

Such a family of maps $\kappa(f), f \in \operatorname{Hom}(\mathcal{Q})$, corresponds precisely to a presheaf $^{22}$ of Hilbert spaces on $\mathcal{Q}$, and this is the most elegant language in which to summarise what we have done so far. Namely, we are constructing representations of the category quantisation monoid, and hence satisfying, Eqs. (61-63) in the following way:

satisfying Eq. (72) is a one-cocycle of the monoid $\operatorname{AF}(\mathcal{Q})$ in its action on $\operatorname{Ob}(\mathcal{Q})$. Furthermore, two multipliers/one-cocycles that are related as in Eq. (74) and with all the $L(A)$, $A \in \mathrm{Ob}(\mathcal{Q})$ being unitary, could be said to differ by a one-coboundary.

${ }^{21}$ This section can be safely ignored at a first reading: a knowledge of presheafs is not essential for the theory being developed.

${ }^{22}$ Here, a presheaf is defined as a contravariant functor from $\mathcal{Q}$ to the category of sets (in our case, Hilbert spaces). 
1. Find a presheaf $\mathcal{K}$ of Hilbert spaces $A \mapsto \mathcal{K}[A]$ over $\mathcal{Q}$. Thus for any arrow $f: A \rightarrow B$ there is a linear map $\kappa(f): \mathcal{K}[B] \rightarrow \mathcal{K}[A]$, and these linear maps satisfy the 'coherence conditions' that if $A \stackrel{\mathrm{g}}{\longrightarrow} B \stackrel{g}{\longrightarrow} C$ then $\mathcal{K}[A] \stackrel{\kappa(f)}{\longleftarrow} \mathcal{K}[B] \stackrel{\kappa(g)}{\longleftarrow} \mathcal{K}[C]$ with $\kappa(f) \kappa(g)=\kappa(g \circ f): \mathcal{K}[C] \rightarrow$ $\mathcal{K}[A]$.

2. Define the quantum states to be cross-sections of the corresponding bundle of Hilbert spaces $\bigcup_{A \in \mathrm{Ob}(\mathcal{Q})} \mathcal{K}[A]$; thus $\psi(A) \in \mathcal{K}[A]$ for all $A \in \mathrm{Ob}(\mathcal{Q})$. The inner product is Eq. (66) for some measure $\mu$ on $\mathrm{Ob}(\mathcal{Q})$.

3. An arrow field $X \in \operatorname{AF}(\mathcal{Q})$ is represented by the operator

$$
(\hat{a}(X) \psi)(A):=\kappa(X(A)) \psi\left(\ell_{X} A\right) .
$$

Functions $\beta: \operatorname{Ob}(\mathcal{Q}) \rightarrow \mathbb{R}$ are represented (in exponentiated form) by the unitary operators

$$
(\hat{V}(\beta) \psi)(A):=e^{-i \beta(A)} \psi(A)
$$

for all $A \in \mathrm{Ob}(\mathcal{Q})$.

The goal is to find a presheaf $\mathcal{K}$ such that the ensuing representation of the category quantisation monoid is irreducible and can separate arrows with the same domain and range.

Note that, although a presheaf structure is a fundamental ingredient in our scheme, it is not the case that the states $\psi$ are defined as sections (or 'global elements') of this presheaf. Indeed, such a section $\psi$ would satisfy the matching conditions

$$
\psi(A)=\kappa(f) \psi(B)
$$

if $f: A \rightarrow B$. This would imply that

$$
(\hat{a}(X) \psi)(A)=\psi(A)
$$

for all arrow fields $X$ and objects $A$. This is why the states are defined to be sections of the bundle of Hilbert spaces associated with the presheaf, rather than sections of the presheaf itself: a section of the bundle does not have to satisfy Eq. (83). 


\subsection{The Adjoint of $\hat{a}(X)$}

\subsubsection{The Simple Case With no Multipliers}

The next step is to find the adjoints of the operators $\hat{a}(X), X \in \operatorname{AF}(\mathcal{Q})$. We start with the simple situation in which there are no multipliers, so that the state vectors are just complex-valued functions on $\operatorname{Ob}(\mathcal{Q})$. We shall also assume initially that the category $\mathcal{Q}$ contains only a finite number of objects. Hence, we can use the inner product

$$
\langle\phi \mid \psi\rangle:=\sum_{A \in \mathrm{Ob}(\mathcal{Q})} \phi(A)^{*} \psi(A)
$$

and then, as usual, for all vectors $|\phi\rangle,|\psi\rangle$ we have

$$
\begin{aligned}
\left\langle\phi\left|\hat{a}(X)^{\dagger}\right| \psi\right\rangle & =\langle\psi|a(X)| \phi\rangle^{*} \\
& =\sum_{A \in \mathrm{Ob}(\mathcal{Q})} \phi\left(\ell_{X} A\right)^{*} \psi(A)
\end{aligned}
$$

To illustrate what this means let us take a simple example of a category with five objects $\left\{A_{1}, A_{2}, A_{3}, B, C\right\}$, and the particular arrow field $X$ defined by

$$
\begin{array}{r}
X\left(A_{1}\right): A_{1} \rightarrow B \\
X\left(A_{2}\right): A_{2} \rightarrow B \\
X\left(A_{3}\right): A_{3} \rightarrow B \\
X(B): B \rightarrow C \\
X(C)=\operatorname{id}_{C}: C \rightarrow C .
\end{array}
$$

Then we have

$$
\left\langle\phi\left|\hat{a}(X)^{\dagger}\right| \psi\right\rangle=\phi(B)^{*}\left(\psi\left(A_{1}\right)+\psi\left(A_{2}\right)+\psi\left(A_{3}\right)\right)+\phi(C)^{*} \psi(B)+\phi(C)^{*} \psi(C)
$$

where the last term comes from the fact that $X(C)=\mathrm{id}_{C}$.

It is clear that, in general, we can write

$$
\left\langle\phi\left|\hat{a}(X)^{\dagger}\right| \psi\right\rangle=\sum_{B \in \mathrm{Ob}(\mathcal{Q})} \sum_{A \in \ell_{X}^{-1}\{B\}} \phi(B)^{*} \psi(A)
$$

where we have been able to sum over all $B \in \mathrm{Ob}(\mathcal{Q})$ by allowing for the fact that $\ell_{X}^{-1}\{B\}$ may be the empty set for some objects $B$. Thus we see that

$$
\left(\hat{a}(X)^{\dagger} \psi\right)(B)=\sum_{A \in \ell_{X}^{-1}\{B\}} \psi(A) .
$$


This result can be extended to the case where the set of objects $\mathrm{Ob}(\mathcal{Q})$ is countably infinite, although the usual care will need to be taken with the domains of the operators $\hat{a}(X)$ and their adjoints.

If Dirac notation is used, we write $\psi(A)$ as $\langle A \mid \psi\rangle$, in which case the equation $(\hat{a}(X) \psi)(A):=\psi\left(\ell_{X} A\right)$ reads $\langle A|\hat{a}(X)| \psi\rangle=\left\langle\ell_{X} A \mid \psi\right\rangle$, and so

$$
\hat{a}(X)^{\dagger}|A\rangle=\left|\ell_{X} A\right\rangle .
$$

In particular, this shows that, for any object $A \in \mathrm{Ob}(\mathcal{Q}), \hat{a}(X)^{\dagger}|A\rangle$ is never zero $^{23}$. In this restricted sense, $\hat{a}(X)^{\dagger}$ looks like a type of creation operator. comes

On the other hand, the equation $\left(\hat{a}(X)^{\dagger} \psi\right)(B)=\sum_{A \in \ell_{X}^{-1}\{B\}} \psi(A)$ be-

$$
\left\langle B\left|\hat{a}(X)^{\dagger}\right| \psi\right\rangle=\sum_{A \in \ell_{X}^{-1}\{B\}}\langle A \mid \psi\rangle
$$

and so

$$
\hat{a}(X)|B\rangle=\sum_{A \in \ell_{X}^{-1}\{B\}}|A\rangle .
$$

In particular, if $B$ is an object that is not the range of any arrow in the arrow field $X$, then $\ell_{X}^{-1}\{B\}=\emptyset$, and hence

$$
\hat{a}(X)|B\rangle=0 .
$$

Thus, in this restricted sense, $\hat{a}(X)$ looks like a type of annihilation operator.

To illustrate these results concretely, let us return to the simple category with five objects $\left\{A_{1}, A_{2}, A_{3}, B, C\right\}$, and the arrow field shown in Eq. (88). Since no arrows in the arrow field $X$ enter $A_{1}, A_{2}$, or $A_{3}$ we have

$$
\hat{a}(X)\left|A_{1}\right\rangle=\hat{a}(X)\left|A_{2}\right\rangle=\hat{a}(X)\left|A_{3}\right\rangle=0 .
$$

On the other hand $\ell_{X}^{-1}\{B\}=\left\{A_{1}, A_{2}, A_{3}\right\}$, and so

$$
\hat{a}(X)|B\rangle=\left|A_{1}\right\rangle+\left|A_{2}\right\rangle+\left|A_{3}\right\rangle .
$$

Finally, $\ell_{X}^{-1}\{C\}=\{B, C\}$ (using $X(C)=\operatorname{id}_{C}$ ), which gives

$$
\hat{a}(X)|C\rangle=|B\rangle+|C\rangle .
$$

\footnotetext{
${ }^{23}$ Of course, this does not exclude the existence of states $|\psi\rangle$ for which $\hat{a}(X)^{\dagger}|\psi\rangle=0$.
} 


\subsubsection{The Arrow Field Operators $\hat{a}(f)$.}

The arrow fields $X_{f}, f \in \operatorname{Hom}(\mathcal{Q})$, are particularly interesting as they generate the arrow fields with finite support. The operators $\hat{a}(f):=\hat{a}\left(X_{f}\right)$ acts as

$$
(\hat{a}(f) \psi)(A)= \begin{cases}\psi(\operatorname{Ran} f) & \text { if Dom } f=A \\ \psi(A) & \text { otherwise }\end{cases}
$$

and so, in Dirac notation,

$$
\hat{a}(f)^{\dagger}|A\rangle= \begin{cases}|\operatorname{Ran} f\rangle & \text { if Dom } f=A \\ |A\rangle & \text { otherwise }\end{cases}
$$

Furthermore, from Eq. (94) we see that

$$
\hat{a}(f)|A\rangle= \begin{cases}|\operatorname{Dom} f\rangle & \text { if } \operatorname{Ran} f=A \\ 0 & \text { otherwise }\end{cases}
$$

Note that the operators $\hat{a}(f)$ and $\hat{a}(f)^{\dagger}$ are always bounded, even when the quantum Hilbert space is infinite dimensional.

\subsubsection{The Situation for a General Measure $\mu$ on $\operatorname{Ob}(\mathcal{Q})$.}

For a general measure $\mu$ on $\operatorname{Ob}(\mathcal{Q})$, if $\hat{a}(X)$ is bounded we have the equation

$$
\left\langle\phi\left|\hat{a}(X)^{\dagger}\right| \psi\right\rangle=\langle\psi|\hat{a}(X)| \phi\rangle^{*}=\int_{\mathrm{Ob}(\mathcal{Q})} d \mu(A) \phi\left(\ell_{X} A\right)^{*} \psi(A) .
$$

If $\phi$ is chosen to be the characteristic function $\chi_{S}$ of a measurable subset $S$ of $\mathrm{Ob}(\mathcal{Q})$, Eq. (102) gives $^{24}$

$$
\begin{aligned}
\int_{S} d \mu(A)\left(\hat{a}(X)^{\dagger} \psi\right)(A) & =\int_{\mathrm{Ob}(\mathcal{Q})} d \mu(A) \chi_{S}\left(\ell_{X} A\right)^{*} \psi(A) \\
& =\int_{\ell_{X}^{-1}[S]} d \mu(A) \psi(A)
\end{aligned}
$$

where $\ell_{X}^{-1}[S]:=\left\{A \in \mathrm{Ob}(\mathcal{Q}) \mid \ell_{X} A \in S\right\}$. Note that if $\operatorname{Ob}(\mathcal{Q})$ is a finite set, and if $\mu$ is the point measure that assigns equal weight 1 to each object $A$, and if we chose $S:=\{B\}$, then the result in Eq. (91) is recovered.

\footnotetext{
${ }^{24}$ Of course, to do this rigourously it is necessary to define precisely what is meant by integrating over vectors.
} 


\subsection{Products of $\hat{a}(X)$ and $\hat{a}(X)^{\dagger}$}

\subsubsection{The Operator $\hat{a}(X)^{\dagger} \hat{a}(X)$.}

When applying this quantum theory to specific physical situations, all important physical quantities must be constructed from the operators $\hat{\beta}, \hat{a}(X)$ and $\hat{a}(X)^{\dagger}$. For example, if the theory is being interpreted canonically, then the Hamiltonian will be particularly important. If the theory is interpreted as a history theory, then the decoherence function will be of central importance. Here we shall look briefly at the quadratic expressions $\hat{a}(X)^{\dagger} \hat{a}(X)$ and $\hat{a}(X) \hat{a}(X)^{\dagger}$.

First, ignoring possible problems with operator domains, left multiplying Eq. (60) with $\hat{a}(X)^{\dagger}$ gives

$$
\hat{a}(X)^{\dagger} \hat{a}(X) \hat{\beta}=\hat{a}(X)^{\dagger} \widehat{\beta \circ \ell_{X}} \hat{a}(X) .
$$

On the other hand, taking the adjoint of Eq. (60) gives $\hat{\beta} \hat{a}(X)^{\dagger}=\hat{a}(X)^{\dagger} \widehat{\beta \circ \ell_{X}}$, and right multiplying this with $\hat{a}(X)$ gives

$$
\hat{\beta} \hat{a}(X)^{\dagger} \hat{a}(X)=\hat{a}(X)^{\dagger} \widehat{\beta \circ \ell_{X}} \hat{a}(X) .
$$

Hence,

$$
\left[\hat{a}(X)^{\dagger} \hat{a}(X), \hat{\beta}\right]=0
$$

which, working on the assumption that the algebra generated by the operators of the form $\hat{\beta}$ is maximal abelian ${ }^{25}$, implies that

$$
\left(\hat{a}(X)^{\dagger} \hat{a}(X) \psi\right)(A)=\alpha_{X}(A) \psi(A)
$$

for some measurable function $\alpha_{X}: \operatorname{Ob}(\mathcal{Q}) \rightarrow \mathbb{R}$.

It is easy to compute $\hat{a}(X)^{\dagger} \hat{a}(X)$ explicitly for the simple case when $\mathcal{Q}$ has a finite number of objects and the inner product Eq. (85) is used. We get

$$
\begin{aligned}
\left(\hat{a}(X)^{\dagger} \hat{a}(X) \psi\right)(A) & =\sum_{C \in \ell_{X}^{-1}\{A\}}(\hat{a}(X) \psi)(C) \\
& =\sum_{C \in \ell_{X}^{-1}\{A\}} \psi\left(\ell_{X} C\right)
\end{aligned}
$$

\footnotetext{
${ }^{25}$ More precisely, assuming that the space $L^{\infty}(\mathrm{Ob}(\mathcal{Q}), d \mu)$ of $\mu$ essentially-bounded realvalued functions on $\mathrm{Ob}(\mathcal{Q})$ is maximal abelian when considered as an algebra of multiplication operators on $L^{2}(\mathrm{Ob}(\mathcal{Q}), d \mu)$.
} 
or, in Dirac notation, $\hat{a}(X)^{\dagger} \hat{a}(X)|A\rangle=\sum_{C \in \ell_{X}^{-1}\{A\}}\left|\ell_{X} C\right\rangle$. However, for each $C \in \ell_{X}^{-1}\{A\}$ we have $\ell_{X} C=A$, and hence

$$
\hat{a}(X)^{\dagger} \hat{a}(X)|A\rangle=\left|\ell_{X}^{-1}\{A\}\right||A\rangle
$$

where $\left|\ell_{X}^{-1}\{A\}\right|$ denotes the number of elements in the set $\ell_{X}^{-1}\{A\}$. As is to be expected, this is consistent with the general result in Eq. (107). ${ }^{26}$

For example, in the model category discussed earlier with the arrow field $X$ in Eq. (88), we have

$$
\hat{a}(X)^{\dagger} \hat{a}(X)|B\rangle=3|B\rangle
$$

\subsubsection{The Operator $\hat{a}(X) \hat{a}(X)^{\dagger}$.}

In a similar way, we can compute

$$
\begin{aligned}
\left(\hat{a}(X) \hat{a}(X)^{\dagger} \psi\right)(A) & =\left(\hat{a}(X)^{\dagger} \psi\right)\left(\ell_{X} A\right) \\
& =\sum_{C \in \ell_{X}^{-1}\left\{\ell_{X} A\right\}} \psi(C)
\end{aligned}
$$

where $\ell_{X}^{-1}\left\{\ell_{X} A\right\}$ is the set of all arrows in the arrow field $X$ whose range is $\ell_{X} A$. In Dirac notation this reads

$$
\hat{a}(X) \hat{a}(X)^{\dagger}|A\rangle=\sum_{C \in \ell_{X}^{-1}\left\{\ell_{X} A\right\}}|C\rangle .
$$

For example, in the model category with five objects $\left\{A_{1}, A_{2}, A_{3}, B, C\right\}$ and the arrow field represented by Eq. (88), we have, for $i=1,2,3$,

$$
\ell_{X}^{-1}\left\{\ell_{X} A_{i}\right\}=\left\{A_{1}, A_{2}, A_{3}\right\}
$$

and so

$$
\hat{a}(X) \hat{a}(X)^{\dagger}\left|A_{i}\right\rangle=\left|A_{1}\right\rangle+\left|A_{2}\right\rangle+\left|A_{3}\right\rangle
$$

for $i=1,2,3$.

\footnotetext{
${ }^{26}$ When there is a general measure $\mu$ on $\mathcal{Q}$ the calculations are more complicated. However, it can be shown that if $\hat{a}(X)$ is a bounded operator, and if $\mu$ is a finite measure, then the Radon-Nikodym derivative $\frac{d \ell_{X} * \mu}{d \mu}$ exists and is equal to the function $\alpha_{X}$ in Eq. (107). Thus, in these circumstances, we have

$$
\left(\hat{a}(X)^{\dagger} \hat{a}(X) \psi\right)(A)=\frac{d \ell_{X *} \mu}{d \mu}(A) \psi(A)
$$

everywhere except on a set of $\mu$-measure zero.
} 


\subsection{Including the Multiplier.}

The calculations become more complicated when the state vectors are Hilbertspace valued with a multiplier $m$. However, the essence is the same, and here we just quote some of the results.

The first is that the adjoint $\hat{a}(X)^{\dagger}$ is given by (c.f. Eq. (91))

$$
\left(\hat{a}(X)^{\dagger} \psi\right)(B)=\sum_{A \in \ell_{X}^{-1}\{B\}} m(X, A)^{\dagger} \psi(A)
$$

where $m(X, A)^{\dagger}: \mathcal{K}[A] \rightarrow \mathcal{K}\left[\ell_{X} A\right]$ is the adjoint ${ }^{27}$ of the linear map $m(X, A):$ $\mathcal{K}\left[\ell_{X} A\right] \rightarrow \mathcal{K}[A]$.

When the category $\mathcal{Q}$ has only a finite number of objects, the operator products $\hat{a}(X) \hat{a}(X)^{\dagger}$ and $\hat{a}(X)^{\dagger} \hat{a}(X)$ can be readily computed. Thus we have (c.f. Eq. (112))

$$
\begin{aligned}
\left(\hat{a}(X) \hat{a}(X)^{\dagger} \psi\right)(A) & =m(X, A)\left[\left(\hat{a}(X)^{\dagger} \psi\right)\left(\ell_{X} A\right)\right] \\
& =\sum_{C \in \ell_{X}^{-1}\left\{\ell_{X} A\right\}} m(X, A) m(X, C)^{\dagger} \psi(C)
\end{aligned}
$$

and (c.f. Eq. (108))

$$
\begin{aligned}
\left(\hat{a}(X)^{\dagger} \hat{a}(X) \psi\right)(A) & =\sum_{C \in \ell_{X}^{-1}\{A\}} m(X, C)^{\dagger}[(\hat{a}(X) \psi)(C)] \\
& =\sum_{C \in \ell_{X}^{-1}\{A\}} m(X, C)^{\dagger} m(X, C) \psi\left(\ell_{X} C\right) \\
& =\sum_{C \in \ell_{X}^{-1}\{A\}} m(X, C)^{\dagger} m(X, C) \psi(A)
\end{aligned}
$$

\subsection{The Question of Irreducibility}

Finally, something should be said about the irreducibility, or otherwise, of these representations of the category quantisation monoid. When quantising a system whose configuration space is a manifold $Q \simeq G / H$, the corresponding quantisation group is the semi-direct product $G \times_{\tau} W$, and the unitary equivalence classes of irreducible representations are classified via induced

\footnotetext{
${ }^{27}$ These adjoint maps $m(X, A)^{\dagger} \equiv \kappa(X(A))^{\dagger}$ define a covariant functor from $\mathcal{Q}$ to the category of sets (in our case, Hilbert spaces).
} 
representation theory in terms of (i) the orbits of $G$ on the dual of $W$, and (ii) the different irreducible representations of $H$ [12].

It remains a task for the future to determine a complete representation theory for the case of a general small category $\mathcal{Q}$; if, indeed, this is possible. However, in the manifold analogy, if $Q$ can be decomposed into more than one $G$-orbit, then there is a corresponding decomposition of the group representation into a direct sum or direct integral. This, at least, should have an analogue in the category case, and so a natural question is whether $\mathrm{Ob}(\mathcal{Q})$ is a single orbit under the action of $\operatorname{AF}(\mathcal{Q})$.

The concept of an 'orbit' is more subtle for an action of a semigroup on a set than it is for a group, and a fuller discussion of this issue is deferred to a later paper in this series. However, on looking at the operators $\hat{a}(X)$ and $\hat{a}(X)^{\dagger}$ as given, for example, in Eq. (92) and Eq. (94) it seems natural to define a subset $O$ of $\mathrm{Ob}(\mathcal{Q})$ to be 'connected' if for any pair of objects $A, B \in O$ there exists a finite collection of objects $\left\{A_{1}, A_{2}, \ldots, A_{N}\right\} \subset O$, with $A_{1}=A, A_{N}=B$ and such that, for all $i=1,2, \ldots N-1$, there exists an arrow with domain $A_{i}$ and range $A_{i+1}$, or an arrow with range $A_{i}$ and domain $A_{i+1}$.

Clearly, if $\mathrm{Ob}(\mathcal{Q})$ decomposes into a disjoint union of connected subsets, then the representation of the category quantisation monoid will decompose in a corresponding way. Thus a necessary condition for the representation to be irreducible is that $\mathrm{Ob}(\mathcal{Q})$ is connected in this sense. All the physical examples we have mentioned so far have this property. However, connectedness alone is certainly not sufficient to guarantee irreducibility, and we will return to this issue later.

\section{Conclusions}

We have seen how to construct a quantum scheme for a system whose configuration space (or history equivalent) is the set of object $\mathrm{Ob}(\mathcal{Q})$ in a small category $\mathcal{Q}$. A key ingredient is the monoid $\operatorname{AF}(\mathcal{Q})$ of arrow fields and its action on $\mathrm{Ob}(\mathcal{Q})$. Multiplier representations are needed to distinguish quantum theoretically between arrows with the same range and domain. Each such representation can be expressed in terms of a presheaf of Hilbert spaces over $\operatorname{Ob}(\mathcal{Q})$.

The material in the present paper is only an introduction to what needs to be done to construct a complete representation theory of a category quantisation monoid. Many topics remain for further research, some of the most 
important of which are the following.

1. A general question is how much the theory can be developed in terms of an arbitrary small category $\mathcal{Q}$, and how much will need to rely on the special properties of particular categories of physical interest.

2. It would be good to determine some general way of specifying the Hilbert spaces $\mathcal{K}[A], A \in \mathrm{Ob}(\mathcal{Q})$, such that the ensuing representation of the category quantisation monoid is both faithful and irreducible. At the very least, this is likely to require a proper study of the meaning, and role, of an orbit of the monoid $\operatorname{AF}(\mathcal{Q})$ as it acts on the $\operatorname{set} \operatorname{Ob}(\mathcal{Q})$. However, it may be that a full discussion of irreducibility can only be given in the context of a case-by-case study with specific categories $\mathcal{Q}$.

3. The classification of inequivalent irreducible representations of the category quantisation monoid will involve the choice of a presheaf of Hilbert spaces, and the choice of the measure $\mu$ used in the inner product in Eq. (66). It is necessary therefore to develop a proper measure theory on the set $\mathrm{Ob}(\mathcal{Q})$. Whether this is feasible for a general small category $\mathcal{Q}$ is unclear, but even if it is, it seems likely that the construction of the physically relevant measures will depend on the details of the category. For example, Brightwell et al have recently developed a particular measure theory on a space of causal sets [14] [15]. This was carried out in the context of constructing a classical stochastic theory of causal sets, but perhaps these are also the correct measures to use in the quantum theory as developed in the present paper?

4. The quantisation of a system whose configuration space is a manifold $Q \simeq G / H$, uses only the finite-dimensional subgroup $G$ of the group of all diffeomorphisms, $\operatorname{Diff}(Q)$, and the question arises therefore if, in the category case, there is some submonoid of $\operatorname{AF}(\mathcal{Q})$ that still acts 'transitively' on $\mathrm{Ob}(\mathcal{Q})$ and which would be a more appropriate entity to use in the category quantisation monoid. The answer is likely to depend strongly on the details of the category $\mathcal{Q}$.

5. In the standard quantum theory of a system whose configuration space is an infinite dimensional topological vector space $V$ (for example, in a quantum field theory), the state vectors are typically functions on the topological dual of $V$ rather than on $V$ itself. This is closely connected to the theory of measures on spaces of this type.

This raises the intriguing question of whether something like this could happen when quantising on a category $\mathcal{Q}$. In other words, are there 
situations in which an analogue of the topological dual is needed; and what is the 'dual' of the set of objects $\mathrm{Ob}(\mathcal{Q})$ ? The answer is likely to be closely linked with the problem of constructing suitable measures on $\mathrm{Ob}(\mathcal{Q})$. It is also related to the issue of whether the quantum scheme should involve only some linear subspace of the set of all realvalued functions on $\mathrm{Ob}(\mathcal{Q})$. This would be an analogue of the use of $W \subset C^{\infty}(Q)$ when $Q \simeq G / H$.

When applied to categories of space-times (or spaces) the scheme described above deals with the quantum states of those structures only. However, in practice, there will be other degrees of freedom too (for example, matter fields), and these need to be incorporated at some point. This could be done by exploiting whatever is known already about the quantisation of these extra degrees of freedom, and adjusting the Hilbert spaces $\mathcal{K}[A]$, $A \in \mathrm{Ob}(\mathcal{Q})$, accordingly. The representation of the category quantisation monoid will then no longer be irreducible because of the presence of these extra modes.

However, it may be possible to include any extra degrees of freedom strictly within the category quantisation scheme by changing the category $\mathcal{Q}$ to get an appropriately extended category quantisation monoid. For example, if $\mathcal{Q}$ is a category of topological spaces, then one might replace the objects (topological spaces) with the spaces of continuous functions on them, with appropriate modifications of the arrows. This would give a type of quantum field theory on a space that is itself quantised.

Note that, if $\mathcal{Q}$ is a category of manifolds, additional degrees of freedom could include quantised metric fields. For example, it would be possible to construct a canonical theory of quantum gravity (in either the traditional formalism, or in the newer scheme based on loop variables) in which the spatial 3-manifold is itself subject to 'quantum fluctuations'. The analogue in a history theory would be to quantise Lorentzian metrics on a quantised background space-time manifold. Or the techniques could be applied to give a version of string theory in which the manifold in which the strings, or $d$-branes, propagate is itself the subject of quantum effects.

Finally, note that, when discussing the quantum theory of causal sets, I have assumed that the space $\mathcal{K}[c]$ associated with each causal set $c$ is a standard Hilbert space, in accordance with normal quantum theory. However, in [16] it is argued that normal quantum theory is problematic in such a situation because the use of the continuum real and complex numbers assumes a priori that the background space and space-time are manifolds, which is not the case if the space-time is a causal set. This suggests that each $\mathcal{K}[c]$ 
should be replaced by something quite different: in fact, by whatever the analogue is for that specific causal set $c$ of the Hilbert space of states in normal quantum theory. It is a task for future research to decide what this may be, but once the decision is made, the techniques described in the present paper would be a good starting point to construct a theory in which the causal sets, and the associated quantum theories, are themselves subject to 'quantum fluctuations'.

These projects are exciting, but it should be emphasised that what is described in the present paper is only a 'tool-kit' for constructing operatorbased models of quantum space-time or space: it needs a creative leap to use these tools to construct a physically realistic model of, for example, quantum causal sets. The key step would be to choose a decoherence functional for the quantum history theory. This decoherence functional would be constructed from the operators described in this paper, but new physical principles are needed to decide its precise form. This is an important topic for future research.

\section{Acknowledgements}

I thank Jeremy Butterfield and Raquel Gracia for a critical reading of the first draft of this paper. Support by the EPSRC in form of grant GR/R36572 is gratefully acknowledged.

\section{References}

[1] L. Bombelli, J. Lee, D. Meyer and R.D. Sorkin, Spacetime as a causal set. Phys. Rev. Lett. 59, 521-524, (1987).

[2] R.D. Sorkin, Spacetime and causal sets. In J.C. D'Olivo, E. NahmadAchar, M. Rosenbaum, M.P. Ryan, L.F. Urrutia and F. Zertuche (eds.), Relativity and Gravitation: Classical and Quantum, (Proceedings of the SILARG VII Conference, held Cocoyoc, Mexico, December, 1990), pages 150-173, World Scientific, Singapore, (1991).

[3] D. P. Rideout and R. D. Sorkin, A classical sequential growth dynamics for causal sets, Phys. Rev. D61, 024002 , (2000).

[4] I. Raptis, Algebraic quantisation of causal sets. Int. Jour. Theor. Phys. 39, 1233, (2000). 
[5] C.J. Isham, Quantum logic and the histories approach to quantum theory. Jour. Math. Phys. 23, 2157-2185, (1994).

[6] C. J. Isham, Topological and global aspects of quantum theory. In Relativity, Groups and Topology II Eds. B. S. DeWitt and R. Stora, (1984).

[7] C. J. Isham, A new approach to quantising space-time: II. Quantising on a category of sets. gr-qc/0304077,(2003).

[8] C. J. Isham, A new approach to quantising space-time: III. State vectors as functions on arrows. (2003).

[9] S. Lang, Algebra. Addison-Wesley Publishing Company, Massachusetts, (1965).

[10] I. Raptis and R. R. Zapatrin, Quantization of discretized spacetimes and the correspondence principle. Int. Jour. Theor. Phys. 39, 1, (2000).

[11] I. Raptis and R. R. Zapatrin, Algebraic description of spacetime foam. Class. Qu. Grav. 20, 4187, (2001).

[12] G. W. Mackey, Unitary Group Representations in Physics, Probability, and Number Theory. Benjamin, Massachusetts, (1978).

[13] A. A. Kirillov, Elements of the Theory of Representations. SpringerVerlag, New York, (1976).

[14] G. Brightwell, H. F. Dowker, R. S. Garcia, J. Henson and R. D. Sorkin, General covariance and the 'problem of time' in a discrete cosmology. In K. G. Bowden (ed.), Correlations: Proceedings of the ANPA 23 Conference, 1-17, August 16-21, Cambridge, England. gr-qc/0202097, (2002).

[15] G. Brightwell, H. F. Dowker, R. S. Garcia, J. Henson and R. D. Sorkin, Observables in causal set cosmology. gr-qc/0210061, (2002).

[16] C. J. Isham, Some reflections on the status of conventional quantum theory when applied to quantum gravity. To appear in Proceedings of the Conference in Honour of Stephen Hawking's 60'th birthday. quantph/0206090, (2003). 